\title{
A MATHEMATICAL PDE PERSPECTIVE ON THE CHAPMAN-ENSKOG EXPANSION
}

\author{
LAURE SAINT-RAYMOND
}

\begin{abstract}
This paper presents in a synthetic way some recent advances on hydrodynamic limits of the Boltzmann equation. It aims at bringing a new light to these results by placing them in the more general framework of asymptotic expansions of Chapman-Enskog type, and by discussing especially the issues of regularity and truncation.
\end{abstract}

The present article is one of two companion papers on the Chapman-Enskog expansion for the Boltzmann equation. While the contribution "Hilbert's 6th Problem: exact and approximate hydrodynamic manifolds for kinetic equations" in this issue of the Bulletin of the American Mathematical Society by A. N. Gorban and I. Karlin 25] aims at extending the Chapman-Enskog method to define a notion of hydrodynamics without any small parameter (that is even far from thermodynamic equilibrium), our contribution focuses on the mathematical justification of the first-order approximations in the fast relaxation limit, and especially on the questions of truncation and regularity. These questions are indeed crucial issues in getting rigorous convergence results starting from renormalized solutions to the Boltzmann equation, which are the only solutions known to exist globally without any restriction on the size of the initial data.

To point out more precisely the difficulties, let us first recall some basic features of the Boltzmann equation which describes the evolution of a perfect gas, represented by its distribution $f \equiv f(t, x, v)$ under the combined effects of free transport and collisions

$$
\begin{aligned}
\partial_{t} f+v \cdot \nabla_{x} f & =Q(f, f) \\
Q(f, f)(v) & =\iint(\underbrace{f\left(v^{\prime}\right) f\left(v_{1}^{\prime}\right)}_{\text {gain }}-\underbrace{f(v) f\left(v_{1}\right)}_{\text {loss }}) b\left(v-v_{1}, \omega\right) d v_{1} d \omega, \\
v^{\prime}=v & +\left(v-v_{1}\right) \cdot \omega \omega, \quad v_{1}^{\prime}=v_{1}-\left(v-v_{1}\right) \cdot \omega \omega .
\end{aligned}
$$

The collision operator $Q$ is quadratic since it accounts for only pairwise elastic interactions. Moreover, it acts only on the $v$-variable insofar as collisions are assumed to be local both in $t$ and $x$. This is the reason why the right-hand side -in general - does not even make sense under the only physical estimates. Formally, the conservations of mass and energy indeed provide

$$
\iint f(t, x, v)\left(1+|v|^{2}\right) d v d x=\iint f_{i n}(x, v)\left(1+|v|^{2}\right) d v d x
$$

Received by the editors July 20, 2013.

2010 Mathematics Subject Classification. Primary 76P05, 35Q20, 35C20. 
whereas the second principle of thermodynamics, also referred to as Boltzmann's $H$-theorem, gives the decay of entropy

$$
\iint f \log f(t, x, v) d x d v+\int_{0}^{t} \int D(f)(s, x) d x d s=\iint f_{i n} \log f_{i n}(x, v) d x d v,
$$

where the entropy dissipation is the nonnegative functional defined by

$$
\begin{aligned}
D(f) & =-\int Q(f, f) \log f d v \\
& =\frac{1}{4} \iint\left(f\left(v^{\prime}\right) f\left(v_{1}^{\prime}\right)-f(v) f\left(v_{1}\right)\right) \log \left(\frac{f\left(v^{\prime}\right) f\left(v_{1}^{\prime}\right)}{f(v) f\left(v_{1}\right)}\right) b d v_{1} d v d \omega .
\end{aligned}
$$

In other words, the collision operator involves the product of two functions of $x$ which are only known to be in some $L \log L$ Orlicz space.

To overcome this difficulty, DiPerna and Lions have introduced in [15] a very weak notion of solution, requiring that $f$ satisfies a family of formally equivalent kinetic equations, obtained by truncation of large tails.

For the sake of simplicity, we will assume here that the collision cross-section $b$, giving the statistical repartition of post-collisional velocities as a function of precollisional velocities and deflection angle $\omega \in S^{2}$, has no singularity, which is the case, for instance, for hard sphere interactions

$$
b\left(v-v_{1}, \omega\right)=\left|\left(v-v_{1}\right) \cdot \omega\right| .
$$

Definition 0.1. A renormalized solution of the Boltzmann equation (0.1) relative to the global equilibrium

$$
M(v)=\frac{1}{(2 \pi)^{3 / 2}} \exp \left(-\frac{1}{2}|v|^{2}\right),
$$

is a function

$$
f \in C\left(\mathbf{R}^{+}, L_{\text {loc }}^{1}\left(\Omega \times \mathbf{R}^{3}\right)\right),
$$

which satisfies in the sense of distributions

$$
\begin{aligned}
& M\left(\partial_{t}+v \cdot \nabla_{x}\right) \Gamma\left(\frac{f}{M}\right)=\Gamma^{\prime}\left(\frac{f}{M}\right) Q(f, f) \quad \text { on } \mathbf{R}^{+} \times \Omega \times \mathbf{R}^{3}, \\
& f_{\mid t=0}=f_{\text {in }} \geq 0 \quad \text { on } \Omega \times \mathbf{R}^{3} .
\end{aligned}
$$

for any $\Gamma \in C^{1}\left(\mathbf{R}^{+}\right)$such that $\left|\Gamma^{\prime}(z)\right| \leq C / \sqrt{1+z}$.

The renormalization process consists in truncating large tails of the distribution function for which the collision operator becomes singular. The crucial remark by DiPerna and Lions is that the condition $\left|\Gamma^{\prime}(z)\right| \leq C /(1+z)$ guarantees that the right-hand side at least makes sense under the only assumptions that the entropy, entropy dissipation, energy, and mass are finite. A later contribution of Lions 32 . shows actually that renormalizations growing like $\Gamma(z)=O(\sqrt{z})$ at infinity are still admissible. Note that the quantity to be studied has the same homogeneity as the wave function in quantum mechanics, which could be considered to be relevant from the physical point of view.

Together with fine tools of analysis (some of which will be used later in this paper for the study of hydrodynamic limits), this renormalization process allows us to prove the existence of global solutions to the Boltzmann equation. 
Theorem 0.2 (DiPerna and Lions). Given any initial data $f_{\text {in }}$ satisfying

$$
H\left(f_{i n} \mid M\right) \equiv \int_{\Omega} \int\left(f_{\text {in }} \log \frac{f_{\text {in }}}{M}-f_{\text {in }}+M\right)(x, v) d v d x<+\infty,
$$

there exists a renormalized solution $f \in C\left(\mathbf{R}^{+}, L_{\text {loc }}^{1}\left(\Omega \times \mathbf{R}^{3}\right)\right)$ relative to $M$ to the Boltzmann equation (0.1) with initial data $f_{i n}$. Moreover, $f$ satisfies the following:

- the continuity equation

$$
\partial_{t} \int f d v+\nabla_{x} \cdot \int f v d v=0
$$

- the global entropy inequality

$$
H(f \mid M)(t)+\int_{0}^{t} \int D(f)(s, x) d s d x \leq H\left(f_{i n} \mid M\right),
$$

where the entropy dissipation $D(f)$ is defined by (0.2).

Note however that, since this notion of solution is very weak, some physical properties, such as the local conservation of momentum and energy or the uniqueness, are not known to hold. We will see that it is an important drawback when considering fluid approximations of the Boltzmann equation.

\section{Asymptotic expansions for the fast Relaxation limit OF THE BOLTZMANN EQUATION}

In the fast relaxation limit, i.e., when the mean free path is very small compared to the typical observation length, we expect the collision process to be dominant and the solution to the Boltzmann equation to be close to local thermodynamic equilibrium. The evolution of the gas should therefore be well approximated by some fluid equations.

1.1. Chapman-Enskog expansion vs Hilbert expansion. The first mathematical studies of hydrodynamic limits of the Boltzmann equation are due to Hilbert [29] on the one hand, and to Chapman and Enskog [12 on the other hand. Note that, in both cases, the derivations are purely formal.

Hilbert's method consists in seeking a formal solution to the scaled Boltzmann equation

$$
\partial_{t} f+v \cdot \nabla_{x} f=\frac{1}{\epsilon} Q(f, f)
$$

with small Knudsen number $\epsilon$, in the form

$$
f(t, x, v, \epsilon)=\sum_{n=0}^{\infty} \epsilon^{n} f_{n}(t, x, v) .
$$

Identifying the coefficients of the different powers of $\epsilon$, we then obtain systems of equations for the successive approximations $f_{0}, f_{0}+\epsilon f_{1}, \ldots$

In particular, at leading order we get

$$
f_{0}(t, x, v) \sim \frac{R_{0}(t, x)}{\left(2 \pi T_{0}(t, x)\right)^{3 / 2}} \exp \left(-\frac{\left|v-U_{0}(t, x)\right|^{2}}{2 T_{0}(t, x)}\right)
$$


for some $R_{0}(t, x), T_{0}(t, x)>0$ and $U_{0}(t, x) \in \mathbf{R}^{3}$. In order that the next order equation can be solved, we further obtain the constraints

$$
\begin{aligned}
\partial_{t} R_{0}+\nabla_{x} \cdot\left(R_{0} U_{0}\right) & =0, \\
\partial_{t}\left(R_{0} U_{0}\right)+\nabla_{x} \cdot\left(R_{0} U_{0} \otimes U_{0}+R_{0} T_{0} I d\right) & =0, \\
\partial_{t}\left(R_{0}\left|U_{0}\right|^{2}+3 R_{0} T_{0}\right)+\nabla_{x} \cdot\left(U_{0}\left(R_{0}\left|U_{0}\right|^{2}+5 R_{0} T_{0}\right)\right) & =0,
\end{aligned}
$$

which are exactly the compressible Euler equations.

Going on in Hilbert's expansion, one gets, as compatibility conditions to solve the hierarchy, that at each order $k \geq 1$, the hydrodynamic part of $f_{k}$ satisfies the linearized compressible Euler equations (with source terms depending on $f_{k-j}$, for $j=1, \ldots, n-1)$. It seems then natural to collect all contributions to the local thermodynamic equilibrium at leading order.

1.1.1. The Ansatz. Such a variant of Hilbert's expansion was found independently by Chapman and Enskog, and is known today as the Chapman-Enskog expansion 12]:

$$
f(t, x, v)=\mathcal{M}_{f}(t, x, v)\left(1+\sum_{k \geq 1} \epsilon^{k} \tilde{g}_{k}(t, x, v)\right),
$$

where $\mathcal{M}_{f}$ is the local Maxwellian with same moments as $f$,

$$
\begin{gathered}
\mathcal{M}_{f}(t, x, v)=\frac{R(t, x)}{(2 \pi T(t, x))^{3 / 2}} \exp \left(-\frac{|v-U(t, x)|^{2}}{2 T(t, x)}\right) \\
R(t, x)=\int f(t, x, v) d v, \quad R U(t, x)=\int v f(t, x, v) d v \\
R\left(|U|^{2}+3 T\right)(t, x)=\int|v|^{2} f(t, x, v) d v
\end{gathered}
$$

and the fluctuations $\tilde{g}_{k}$ are functions of $v$ depending on $(t, x)$ through $R(t, x)$, $U(t, x)$, and $T(t, x)$, and their partial $x$-derivatives evaluated at $(t, x)$. Note that, at variance with Hilbert's expansion, the Chapman-Enskog Ansatz requires knowing in advance that the successive corrections to the compressible Euler system (1.1) within any order in $\epsilon$ are systems of local conservation laws.

The first correction to the compressible Euler equations is then given by

$$
\partial_{t} \mathcal{M}_{f}+v \cdot \nabla_{x} \mathcal{M}_{f}=-\mathcal{M}_{f} \mathcal{L}_{\mathcal{M}_{f}}\left(\tilde{g}_{1}\right),
$$

or equivalently

$$
\begin{array}{r}
\partial_{t}\left(\log R-\frac{3}{2} \log T-\frac{1}{2 T}|v-U|^{2}\right)+\nabla_{x}\left(\log R-\frac{3}{2} \log T-\frac{1}{2 T}|v-U|^{2}\right) \cdot v \\
=-\mathcal{L}_{\mathcal{M}_{f}}\left(\tilde{g}_{1}\right),
\end{array}
$$

where $\mathcal{L}_{\mathcal{M}_{f}}$ denotes the linearization of the collision operator at the local Maxwellian $\mathcal{M}_{f}$

$$
\mathcal{L}_{\mathcal{M}_{f}} g=-\frac{1}{\mathcal{M}_{f}} Q\left(\mathcal{M}_{f}, \mathcal{M}_{f} g\right)-\frac{1}{\mathcal{M}_{f}} Q\left(\mathcal{M}_{f} g, \mathcal{M}_{f}\right)
$$


Then, using the properties of the linearized collision operator $\mathcal{L}_{\mathcal{M}_{f}}$, namely the fact that it is a Fredholm operator, one obtains the weakly viscous Navier-Stokes system with $O(\epsilon)$ dissipation terms

$$
\begin{gathered}
\partial_{t} R+\nabla_{x} \cdot(R U)=0, \\
\partial_{t}(R U)+\nabla_{x} \cdot(R U \otimes U+R T I d)=\epsilon \nabla_{x} \cdot(\mu(R, T) D(U))+O\left(\epsilon^{2}\right), \\
\partial_{t}\left(R|U|^{2}+3 R T\right)+\nabla_{x} \cdot\left(U\left(R|U|^{2}+5 R T\right)\right) \\
=\epsilon \nabla_{x} \cdot\left(\kappa(R, T) \nabla_{x} T\right)+\epsilon \nabla_{x} \cdot(\mu(R, T) D(U) \cdot U)+O\left(\epsilon^{2}\right),
\end{gathered}
$$

where $D(U)$ denotes the traceless part of the deformation tensor

$$
D(U)=\frac{1}{2}\left(\nabla_{x} U+\left(\nabla_{x} U\right)^{T}\right)-\frac{1}{3}\left(\nabla_{x} \cdot U\right) I d,
$$

and the diffusive coefficients, namely the viscosity $\mu \equiv \mu(R, T)$ and the heat conductivity $\kappa \equiv \kappa(R, T)$, are defined in terms of the linearized collision operator $\mathcal{L}_{\mathcal{M}_{f}}$.

We then deduce formally that the solution to the weakly viscous Navier-Stokes equations is close to the moments of the solution $f$ to the the Boltzmann equation at order $O\left(\epsilon^{2}\right)$.

1.1.2. A better notion of projection on the slow manifold. Let us now compare both asymptotic expansions. On the one hand, the Chapman-Enskog expansion could seem a little bit mysterious insofar as one needs to know in advance that the thermodynamic fields will satisfy a closed system at any order. On the other hand, it is quite natural in view of the scale separation in the equation

$$
\partial_{t} f+v \cdot \nabla_{x} f=\frac{1}{\epsilon} Q(f, f),
$$

which suggests some splitting between the local relaxation encoded by the homogeneous equation

$$
\partial_{t} f=\frac{1}{\epsilon} Q(f, f)
$$

and the transport on macroscopic time and space scales.

The hypocoercive mechanism relating the effects of transport and collisions has been studied in detail - especially by Desvillettes and Villani [14] - to describe the long time behaviour of a gas governed by the Boltzmann equation when collisions and transport are phenomena of the same order. The idea is that the entropy can be decomposed as a sum of a "purely kinetic part" and a "macroscopic entropy":

$$
\int f \log f d v=\int\left(f \log \frac{f}{\mathcal{M}_{f}}-f+\mathcal{M}_{f}\right) d v+\int \mathcal{M}_{f} \log \mathcal{M}_{f} d v .
$$

In the present scaling, assuming - according to Cercignani's conjecture - that the entropy dissipation controls the purely kinetic part of the entropy (which is not correct for the Boltzmann equation, but holds for instance for its BGK (Bathnagar, Gross, and Krook) approximation), we see that the purely kinetic part of the entropy will relax exponentially to 0 as $\epsilon \rightarrow 0$, while the macroscopic part is expected to have a slow evolution.

The splitting between the hydrodynamic part and the purely kinetic part also corresponds to what would be done in the case of a linear singular perturbation problem: projection on the kernel of the singular perturbation to get the constraint 
at leading order, then projection on the orthogonal subspace to get the slow evolution. This will be exactly the strategy we will use when dealing with fluctuations to get incompressible hydrodynamic limits (see Section 2).

Remark 1.1. Note however that such a heuristic analysis relies on the fact that $\left(\partial_{t}+v \cdot \nabla_{x}\right)$ behaves as a bounded operator, which can be justified only when considering smooth functions.

1.1.3. A systematic method to get higher-order fluid approximations. Iterating this process gives a systematic way of deriving equations of hydrodynamics having formally any given order of accuracy with respect to the Knudsen number $\epsilon$. The crucial property is the fact that, under suitable assumptions on the collision crosssection (satisfied in particular for hard-sphere interactions), for all Maxwellians $\mathcal{M}$, the linearized collision operator $\mathcal{L}_{\mathcal{M}}$ satisfies the Fredholm alternative (see [29], 26] for the proof)

(i) $\mathcal{L}_{\mathcal{M}} g=0$ if and only if $g \in \operatorname{Ker}\left(\mathcal{L}_{\mathcal{M}}\right)=\operatorname{span}\left(1, v,|v|^{2}\right)$,

(ii) $\mathcal{L}_{\mathcal{M}}$ is self-adjoint and $\int g \mathcal{L}_{\mathcal{M}} g \mathcal{M} d v \geq C\|g-\Pi g\|_{L^{2}(\mathcal{M} \nu d v)}^{2}$,

(iii) $\operatorname{Ker}\left(\mathcal{L}_{\mathcal{M}}\right) \oplus R\left(\mathcal{L}_{\mathcal{M}}\right)=L^{2}(\mathcal{M} \nu d v)$,

denoting by $\pi$ the orthogonal projection on $\operatorname{Ker}\left(\mathcal{L}_{\mathcal{M}}\right)$, and by $\nu$ the collision frequency

$$
\nu(v)=\iint \mathcal{M}_{1} b\left(v-v_{1}, \omega\right) d v_{1} d \omega .
$$

Let us introduce the following decomposition (referred to in the sequel as the Chapman-Enskog decomposition)

$$
f_{\epsilon}=M_{\epsilon}+\epsilon h_{\epsilon},
$$

where the moments $R_{\epsilon}, U_{\epsilon}, T_{\epsilon}$ defining $M_{\epsilon}$ are the "true" hydrodynamic moments of $f_{\epsilon}$. The equation for the purely kinetic part then states

$$
h_{\epsilon}=h_{0}+\epsilon \frac{M_{\epsilon}}{R_{\epsilon}} \mathcal{L}_{\epsilon}^{-1}\left(\frac{\left(\partial_{t}+v \cdot \nabla_{x}\right) h_{\epsilon}-Q\left(h_{\epsilon}, h_{\epsilon}\right)}{M_{\epsilon}}\right),
$$

where

$$
\begin{aligned}
h_{0} & =\frac{M_{\epsilon}}{R_{\epsilon}} \mathcal{L}_{\epsilon}^{-1}\left(\left(\partial_{t}+v \cdot \nabla_{x}\right) \log M_{\epsilon}\right) \\
& =M_{\epsilon}\left(\frac{1}{R_{\epsilon} T_{\epsilon}} \nabla_{x} U_{\epsilon}: \mathcal{L}_{\epsilon}^{-1} \Phi_{\epsilon}+\frac{1}{2 R_{\epsilon} T_{\epsilon}^{2}} \nabla_{x} T_{\epsilon} \cdot \mathcal{L}_{\epsilon}^{-1} \Psi_{\epsilon}\right), \\
& \text { with } \Phi_{\epsilon}=\left(v-U_{\epsilon}\right)^{\otimes 2}-\frac{1}{3}\left|v-U_{\epsilon}\right|^{2} I d, \quad \Psi_{\epsilon}=\frac{1}{2}\left(v-U_{\epsilon}\right)\left(\left|v-U_{\epsilon}\right|^{2}-5\right) .
\end{aligned}
$$

Here and in the sequel we denote by $\mathcal{L}_{\epsilon}$ the linearized collision operator at $M_{\epsilon}$, and by $\mathcal{L}_{\epsilon}^{-1}$ its inverse on $\operatorname{Ker}\left(\mathcal{L}_{\epsilon}\right)^{\perp}$ extended by 0 on $\operatorname{Ker}\left(\mathcal{L}_{\epsilon}\right)$.

We can therefore compute the successive orders of the approximation just by inverting $\mathcal{L}_{\epsilon}$ :

$$
h_{n+1}=\frac{M_{\epsilon}}{R_{\epsilon}} \mathcal{L}_{\epsilon}^{-1}\left(\frac{\left(\partial_{t}+v \cdot \nabla_{x}\right) h_{n-1}-\sum_{j=0}^{n-1} Q\left(h_{j}, h_{n-1-j}\right)}{M_{\epsilon}}\right) .
$$


The general equations of hydrodynamics then state

$$
\int\left(\begin{array}{c}
1 \\
v \\
|v|^{2}
\end{array}\right)\left(\partial_{t}+v \cdot \nabla_{x}\right) M_{\epsilon} d v+\epsilon \int\left(\begin{array}{c}
1 \\
v \\
|v|^{2}
\end{array}\right) \nabla_{x} \cdot\left(\left(v-U_{\epsilon}\right) \sum_{n=0}^{+\infty} \epsilon^{n} h_{n}\right) d v=0 .
$$

\subsection{The truncation issue.}

1.2.1. Some ill-posed fluid models. The next step after the Navier-Stokes equations gives the so-called Burnett equations. At this stage, the respective corrections for the momentum and temperature equations are given by

$$
\int h_{1} \Phi_{\epsilon} d v \text { and } \int h_{1} \Psi_{\epsilon} d v
$$

A tedious but straightforward computation (given for instance in [13] or [5]) shows that, up to terms with lower derivatives, one has

$$
\begin{aligned}
\partial_{t} U_{\epsilon} & =\frac{2}{3} \frac{\epsilon^{2}}{R_{\epsilon}^{2}}\left(A \frac{T_{\epsilon}}{R_{\epsilon}} \Delta_{x} \nabla_{x} R_{\epsilon}+(A-C) \Delta_{x} \nabla_{x} T_{\epsilon}\right), \\
\partial_{t} T_{\epsilon} & =\frac{4}{9} \frac{\epsilon^{2}}{R_{\epsilon}^{2}}\left(T_{\epsilon}(B-C) \Delta \nabla_{x} \cdot U_{\epsilon}\right),
\end{aligned}
$$

for some coefficients $A, B, C$ depending only on the molecular models for the Boltzmann equation.

The point is that the hyperbolicity condition is generally not satisfied by the principal symbol. In the one-dimensional case, the nonzero eigenvalues are indeed given by

$$
\lambda_{ \pm}= \pm\left(\frac{2}{3} T_{\epsilon}(B-C)(A-C)\right)^{1 / 2}
$$

which are not real if $(B-C)(A-C)<0$ and which holds for instance in the case of hard-sphere interactions (see [5] for estimates of the coefficients $A, B$, and $C$ ). These formulas therefore show that the Burnett equations become unstable with respect to short-wave perturbations, or in other words that they are ill-posed, which is a first obvious obstacle to getting rigorous approximations beyond the NavierStokes level. The amplification of short acoustic waves indeed contradicts the $H$ theorem, since all near-equilibrium perturbations should decay. The situation does not improve in the next, super-Burnett approximation.

This obstacle may however be circumvented by a truncation process, to be compared with normal form techniques for dynamical systems. The idea is to modify the equations

$$
x_{t}+i\left(B_{0}+\epsilon^{2} B_{1}\right) x+\epsilon A_{0} x=O\left(\epsilon^{3}\right)
$$

obtained by the Chapman-Enskog expansion, according to some change of variable

$$
z=x+\epsilon^{2} R x
$$

so that

$$
z_{t}+i\left(B_{0}+\epsilon^{2} \tilde{B}_{1}\right) z+\epsilon A_{0} z=O\left(\epsilon^{3}\right),
$$

where $\tilde{B}_{1}=B_{1}+\left(R B_{0}-B_{0} R\right)$ is real and symmetric.

Such a class of transformation (preserving a form of conservation laws) has been exhibited by Bobylev [6]. 
1.2.2. A divergent series. Admitting that one can reproduce such a regularization process at any order, we end up with a family of locally well-posed fluid equations. A natural question is then to understand what is the meaning of this formal series. Note that exact summations of the Chapman-Enskog expansion are known only in a very limited number of cases 28 . Thus, in general, such an asymptotic expansion is only formal and has to be understood in the same way as in semiclassical analysis.

Considering smooth functions, the correction terms in the hydrodynamic equations are expected to be small in the limit $\epsilon \rightarrow 0$ (see Remark 1.1), which tells us something about the consistency of the approximation. In order to prove that the solutions to these approximate equations are close to the "true" moments of the Boltzmann equation at some fixed order in $\epsilon$, we further need some stability, which is of course the most difficult part from the mathematical point of view.

We insist here that there are actually two additional difficulties:

- stability is required to deduce an asymptotic expansion of the solution from the asymptotic expansion of the equation;

- but even consistency is conditioned to the fact that the functions to be considered are smooth, which is the matter of the next section.

1.3. The regularity issue. From the mathematical point of view, the understanding of the fast relaxation limit falls short of these questions of higher-order approximations. Even the first two orders of the Chapman-Enskog expansion-which can be caught easily at the formal level - still lack for a rigorous derivation.

1.3.1. No uniform regularity estimate. The main difficulty is probably related to the regularity issue, insofar as the compressible Euler equations are not known to be globally well-posed even for small data. More precisely, we expect singularities to develop in finite time, so that classical solutions will generically blow up 41 .

In the one-dimensional case, we have a much more precise description of these singularities and of their propagation, at least under an additional criterion of entropy [35]. The question of knowing whether this admissibility criterion is the relevant one in the fast relaxation limit is still largely open, and it will be discussed at the end of the paper following [42.

Anyway, since the solutions of the first-order hydrodynamic approximation exhibit singularities, such as shocks or discontinuities, the question of their stability seems out of reach at the present time. There are however some very partial results in this direction, proving that some self-similar profiles, namely one-dimensional rarefaction waves [11] and one-dimensional shocks [36], can be obtained as the limit of some suitable families of solutions to the Boltzmann equation in the fast relaxation limit $\epsilon \rightarrow 0$.

Note that these stability results are still quite far from their counterpart for one-dimensional weakly viscous perturbations [9], since they cannot deal with superposition (Riemann problem) and a fortiori interactions of these elementary waves (Glimm's scheme). The key argument in [9] is a central manifold result giving some stability with little regularity. More precisely it requires only the nonuniform regularity estimate coming from the viscous dissipation as well as some uniform BV estimate.

1.3.2. Dissipation and averaging lemma. Let us therefore investigate what kind of regularity can be expected for the Boltzmann equation in the fast relaxation limit. We will describe here the main mechanisms providing regularity in order to get a 
qualitative picture of the different terms in the expansion, but we insist that some arguments are not completely correct. More precise and rigorous results will be given in the next part when proving convergence theorems.

We recall that we are interested in describing the solution to

$$
\partial_{t} f+v \cdot \nabla_{x} f=\frac{1}{\epsilon} Q(f, f)
$$

in the limit $\epsilon \rightarrow 0$, and that the only uniform a priori bounds we can use are those coming from the entropy inequality

$$
H(f \mid M)(t)+\frac{1}{\epsilon} \int_{0}^{t} \int D(f)(s, x) d s d x \leq H\left(f_{\text {in }} \mid M\right),
$$

assuming that the initial entropy is finite.

We have first to note that the regularity issue arises not only when considering the fast relaxation limit. It is also crucial when proving the weak stability of the Boltzmann equation and establishing the existence of renormalized solutions (cf Theorem 0.2). The stability of the collision term $\Gamma^{\prime}(f / M) Q(f, f)$ indeed requires some strong compactness on the moments of $f$, inherited from some uniform regularity estimate. The key argument here is the following averaging lemma [17.

Theorem 1.2 (Golse, Lions, Perthame, and Sentis). Let $f \equiv f(t, x, v)$ be such that $f$ and $\left(\partial_{t}+v \cdot \nabla_{x}\right) f$ both belong to $L^{p}\left(\mathbf{R} \times \mathbf{R}^{d} \times \mathbf{R}^{d}\right)$. Then, for all $\varphi \in C_{c}\left(\mathbf{R}^{d}\right)$, the moment $\int f(t, x, v) \varphi(v) d v$ belongs to $W^{s, p}\left(\mathbf{R} \times \mathbf{R}^{d}\right)$ with $0<s<\inf \left(\frac{1}{p}, 1-\frac{1}{p}\right)$.

In the fast relaxation limit, we obtain that

$$
\begin{aligned}
M\left(\partial_{t} \Gamma(f / M)+v \cdot \nabla_{x} \Gamma(f / M)\right) \\
=\frac{1}{\epsilon} \Gamma^{\prime}(f / M) Q(f, f) \\
=\Gamma^{\prime}(f / M) \iint\left(\frac{\sqrt{f^{\prime} f_{1}^{\prime}}-\sqrt{f f_{1}}}{\sqrt{\epsilon}}\right)^{2} b d v d v_{1} d \omega \\
\quad+\frac{1}{\sqrt{\epsilon}} \sqrt{f} \Gamma^{\prime}(f / M) \iint \sqrt{f_{1}}\left(\frac{\sqrt{f^{\prime} f_{1}^{\prime}}-\sqrt{f f_{1}}}{\sqrt{\epsilon}}\right) b d v d v_{1} d \omega
\end{aligned}
$$

from which we deduce that

$$
\left\|\int \Gamma(f / M) \phi M d v\right\|_{W_{t, x}^{s, 1}}=O\left(\epsilon^{-\frac{s}{2}}\right)
$$

for $s$ small enough. This tells us more or less that the frequencies are localized in a sphere of diameter $\epsilon^{-1 / 2}$.

1.3.3. What about higher-order regularity? The previous interpretation, however, is not correct because the cancellation occuring at leading order due to the entropy dissipation bound has no counterpart at higher orders. Even at the formal level, we have

$$
\partial_{t} D_{t, x} f+v \cdot \nabla_{x} D_{t, x} f=\frac{2}{\epsilon} Q\left(D_{t, x} f, f\right)
$$

so that, even with a $L^{\infty}$ bound on $f$ and good hypoelliptic properties of the transport operator, we will get a loss of $O\left(\frac{1}{\epsilon}\right)$ for each additional derivative.

These remarks are crucial in view of Remark 1.1. which states that one of the underlying assumptions in the Chapman-Enskog expansion is the fact that the 
operator $\left(\partial_{t}+v \cdot \nabla_{x}\right)$ behaves as a bounded operator. We indeed note that the actual expansion parameter is $\epsilon \nabla_{x}$, which is not small if functions have derivatives of order $O\left(\frac{1}{\epsilon}\right)$.

1.4. Extrapolation of the Chapman-Enskog procedure in the absence of scale separation. The previous iterative scheme leads to a formal series of operators governing the hydrodynamic part of the motion, which can be viewed as the projection of the motion on some invariant manifold. If such a manifold exists and if it is analytic, it should be obtained from the Chapman-Enskog germ near local equilibria by unique analytic continuation. However, this does not guarantee the convergence of the series in powers of $\epsilon \nabla_{x}$ (especially at high frequencies).

In order to bypass this difficulty, Gorban and Karlin have proposed in [23, 24, a slightly different strategy, replacing the iterative construction of the invariant manifold by a more direct approach which does not need any scale separation between transport and relaxation: they actually use the symmetries and invariances of the microscopic system to design a suitable ansatz. This approach has been applied successfully to some simplified linear models with finite numbers of moments. The idea is to consider minimal kinetic models where the Chapman-Enskog method can be studied exactly, thereby providing the basis to compare various approximations in extending the hydrodynamic description beyond the Navier-Stokes approximation.

In the linear case [23, the Chapman-Enskog series is summed up exactly in closed form. This result shows that the corresponding hydrodynamics, despite divergences of some truncated expansions, is globally well defined because of dispersive effects. This indicates in particular that the Knudsen number $\epsilon$ used to get the expansion is maybe not the good parameter: finite-order truncations do not seem to provide suitable approximations in the whole, and especially in the short-wave, domain. As noted by Slemrod [42, this could mean that even the Navier-Stokes approximation is wrong in the vicinity of compressible shocks.

In the nonlinear case, there is no immediate generalization of these techniques. Karlin and Gorban [24 have thus introduced reduced descriptions based on the principle of dynamic invariance. They sum exactly a subseries of the ChapmanEnskog expansion, for instance the dominant contribution in the limit of high average velocities.

The main drawback of this approach comes from the fact that, like the classical perturbative Chapman-Enskog expansion, the extended Chapman-Enskog procedure assumes the existence of the invariant manifold, which is not known in general and especially for nonlinear systems. Thus, at this stage, this systematic - even promising - strategy does not say anything about the hydrodynamics associated to the full nonlinear Boltzmann equation. Note however that the approach of Liu and $\mathrm{Yu}$ [36] of the compressible limit is in the same spirit and could be probably reinterpreted in such terms if at least the existence of the invariant manifold was known.

\section{Fluctuations around a global equilibrium AND INCOMPRESSIBLE HYDRODYNAMIC LIMITS}

A natural idea to avoid these complicated questions about the compressible Euler equations is to consider fluctuations around some special solutions, the simplest ones being constant solutions. At the present time, this is essentially the only mathematical framework in which we have quite satisfactory results describing the 
fast relaxation limit. Note that this framework is not so restrictive insofar as in the long time limit (in particular on diffusive or dispersive time scales), all solutions of the compressible Euler equations should behave as constants at least locally.

At this stage, there is no problem with the leading order approximation, which is simply a constant state, say without loss of generality

$$
M(v)=\frac{1}{(2 \pi)^{3 / 2}} \exp \left(-\frac{|v|^{2}}{2}\right) .
$$

In particular, it is smooth and stable thanks to Boltzmann's $H$-theorem stating that the entropy is a Lyapunov functional for the Boltzmann dynamics

$$
H(f \mid M)(t) \equiv \iint\left(f \log \frac{f}{M}-f+M\right)(t, x, v) d x d v \leq H\left(f_{i n} \mid M\right) .
$$

2.1. A problem of convergence rather than asymptotics. A first simplification in this setting is that we can define easily the fluctuation

$$
M g_{\epsilon}=\frac{1}{\delta_{\epsilon}}\left(f_{\epsilon}-M\right)
$$

with the proper scaling $\delta_{\epsilon}$ such that the first correction to the Euler equations now appears as a term of order 1 .

From the formal expansion, we deduce that convection and diffusion terms will be comparable if $\delta_{\epsilon} \sim \epsilon$. This corresponds to having the Mach and Knudsen numbers of the same order of magnitude, which is relevant with the Von Karman relation for perfect gases giving the Reynolds number as the ratio of the Mach and Knudsen numbers. Of course, to observe a macroscopic motion, we further need to dilate time by a factor $1 / \epsilon$.

We therefore end up with the scaled equation

$$
\epsilon \partial_{t} g_{\epsilon}+v \cdot \nabla_{x} g_{\epsilon}=-\frac{1}{\epsilon} \mathcal{L} g_{\epsilon}+\mathcal{Q}\left(g_{\epsilon}, g_{\epsilon}\right)
$$

denoting by $\mathcal{L}$ the linearized collision operator at $M$ and by $\mathcal{Q}$ the quadratic part of the collision operator

$$
\mathcal{Q}(g, g)=\frac{1}{M} Q(M g, M g) .
$$

With these notations and scalings, justifying the second-order Chapman-Enskog expansion then comes down to proving the convergence of $\left(g_{\epsilon}\right)$ to an infinitesimal Maxwellian, the moments of which should satisfy the incompressible Navier-Stokes equations. This is of course much simpler than dealing with a multiscale expansion, especially because we do not need to control the error in some strong topology.

To make this remark more comprehensive, let us just go through a small digression and recall the different types of methods which are usually used to deal with such singular perturbation problems.

- Energy or entropy methods are based on some strong (or weak-strong) stability principle: the idea is to control some distance between the solution of the singular perturbation problem and its (possibly multiscale) approximation. In particular, such methods provide a convergence rate. But as a counterpart, they generally ask for some regularity, typically Lipschitz regularity at least on the limiting field, to control the growth of this distance with respect to time. 
- Weak stability methods require in general many fewer assumptions, but can only describe a weak limit without any precise information on the convergence (only one scale, no description of possible oscillations, no rate of convergence, ....).

Note that both approaches are conditioned by the structure of the limiting system and its stability properties.

2.2. A well-posed limiting model. The second important feature of the regime we consider here is that the limiting system

$$
\partial_{t} u+\left(u \cdot \nabla_{x}\right) u+\nabla_{x} p=\mu \Delta_{x} u, \quad \nabla_{x} \cdot u=0,
$$

is well-posed and stable. Actually it is both

- locally well-posed in the sense of Hadamard for smooth initial data, say in $H^{s}$ with $s>\frac{5}{2}$, and then strongly stable;

- and globally well-posed in a weaker sense (not implying uniqueness), and weakly stable.

This second notion of solution fits better with physics insofar as the initial data is only required to satisfy energy bounds. We therefore expect that it should provide a good framework to study the transition between kinetic and fluid scales.

Remark 2.1. Note that historically the first derivation of the incompressible NavierStokes equations from the scaled Boltzmann equation has been obtained in the framework of smooth solutions [4, but the result is not optimal in the sense that smallness and regularity are imposed on the initial data, that only well-prepared profiles and thermodynamic fields can be considered, and that boundary conditions cannot be dealt with using a simple extension of the method.

Actually what is proved in [4] and the subsequent literature is rather the existence of one family of solutions to the scaled Boltzmann equation having the expected asymptotic behaviour rather than a characterization of all limiting points in the fast relaxation limit.

We will therefore focus here on global weak solutions satisfying the Leray energy inequality

$$
\|u(t)\|_{L^{2}(\Omega)}^{2}+2 \mu \int_{0}^{t}\left\|\nabla_{x} u(s)\right\|_{L^{2}(\Omega)}^{2} d s \leq\left\|u_{i n}\right\|_{L^{2}(\Omega)}^{2},
$$

and which are stable under weak convergence [31.

Theorem 2.2 (Leray). Let $u_{i n} \in L^{2}(\Omega)$ be a divergence-free vector field. Then there exists (at least) one global weak solution $u \in L_{\mathrm{loc}}^{2}\left(\mathbf{R}^{+}, H^{1}(\Omega)\right) \cap C\left(\mathbf{R}^{+}, w-\right.$ $L^{2}(\Omega)$ ) to the incompressible Navier-Stokes equations (2.2). It further satisfies the energy inequality (2.3).

The dissipation term in (2.3) provides indeed some spatial regularity which, combined with the time regularity coming from the evolution equation in (2.2), gives some strong compactness and therefore stability of the (nonlinear) convection term.

Remark 2.3. This weak stability result can be extended without any additional difficulty to the full viscous incompressible approximation of the Boltzmann equation

$$
\begin{aligned}
\partial_{t} u+\left(u \cdot \nabla_{x}\right) u+\nabla_{x} p=\mu \Delta_{x} u, & \nabla_{x} \cdot u & =0, \\
\partial_{t} \theta+\left(u \cdot \nabla_{x}\right) \theta=\kappa \Delta \theta, & \nabla_{x}(\rho+\theta) & =0,
\end{aligned}
$$


since the equation for the temperature is a simple linear transport-diffusion equation, once the convection field $u \in L_{\text {loc }}^{2}\left(\mathbf{R}^{+}, H^{1}(\Omega)\right) \cap C\left(\mathbf{R}^{+}, w-L^{2}(\Omega)\right)$ is known.

A similar mechanism will give the weak convergence of the thermodynamic fields associated to the scaled Boltzmann equation as the Knudsen and Mach number goes to 0 at the same rate. In such a viscous regime, the Leray energy inequality (2.3) and the DiPerna-Lions entropy inequality (0.6) are indeed very similar objects.

2.3. Entropy and entropy-dissipation estimates. The entropy inequality

$$
H\left(f_{\epsilon} \mid M\right)+\frac{1}{\epsilon^{2}} \int_{0}^{t} \int D\left(f_{\epsilon}\right)(s, x) d x d s \leq H\left(f_{\epsilon}^{i n} \mid M\right)
$$

is the central tool of the asymptotic analysis, exactly as its limiting form - the Leray energy inequality - in the theory of weak solutions to the incompressible NavierStokes equations.

2.3.1. Control of the fluctuation. In order that the scaling assumption is consistent, we consider initial data which are perturbations of order $\epsilon$ around $M$, for instance

$$
f_{\epsilon}^{i n}=M\left(1+\epsilon g^{i n}\right)
$$

so that

$$
H\left(f_{\epsilon}^{i n} \mid M\right) \leq C_{0} \epsilon^{2} .
$$

We indeed check that, for such distributions, the thermal speed is typically of order 1 while the bulk velocity is of order $\epsilon$, which guarantees that the Mach number is effectively of order $\epsilon$.

The first use of the scaled relative entropy inequality is to ensure that the solution to the Boltzmann equation remains a perturbation of order $\epsilon$ around $M$ for all times, that is to give some uniform a priori estimate on the fluctuation $g_{\epsilon}$ defined by

$$
f_{\epsilon}=M\left(1+\epsilon g_{\epsilon}\right) .
$$

The relative entropy can be indeed recast in the form

$$
\frac{1}{\epsilon^{2}} H\left(f_{\epsilon} \mid M\right)=\frac{1}{\epsilon^{2}} \iint M h\left(\epsilon g_{\epsilon}\right) d v d x \quad \text { with } \quad h(z)=(1+z) \log (1+z)-z .
$$

Since $h(z) \sim z^{2} / 2$ as $z \rightarrow 0$, we thus expect the scaled relative entropy to almost control the $L^{2}$-norm of the fluctuation $g_{\epsilon}$

$$
\iint M g_{\epsilon}^{2} d v d x \leq \frac{C\left(\left\|\epsilon g_{\epsilon}\right\|_{\infty}\right)}{\epsilon^{2}} H\left(f_{\epsilon} \mid M\right) .
$$

Nevertheless, for fixed $\epsilon$, we have only a $L^{1}$-bound. It comes for instance from Young's inequality

which provides

$$
M\left|g_{\epsilon}\right| \leq \frac{1}{\epsilon^{2}}\left(M h\left(\epsilon g_{\epsilon}\right)+M h^{*}(\epsilon)\right),
$$

$$
\left\|g_{\epsilon}\right\|_{L^{1}(M d v)}=O(1)_{L_{t}^{\infty}\left(L_{x}^{1}\right)}+O(1)_{L_{t, x}^{\infty}} .
$$

Refined estimates can be obtained by introducing the renormalized fluctuation

$$
\hat{g}_{\epsilon}=\frac{2}{\epsilon}\left(\sqrt{1+\epsilon g_{\epsilon}}-1\right) .
$$

A simple functional inequality indeed shows that

$$
\left|\hat{g}_{\epsilon}^{2}\right| \leq \frac{2}{\epsilon^{2}} h\left(\epsilon g_{\epsilon}\right)
$$


so that

$$
\left\|\hat{g}_{\epsilon}\right\|_{L^{2}(M d v)}=O(1)_{L^{2}(d x)} .
$$

Note that the identity

$$
g_{\epsilon}=\hat{g}_{\epsilon}+\frac{\epsilon}{4} \hat{g}_{\epsilon}^{2}
$$

allows us, in particular, to retrieve the $L^{1}$-bound (2.5) on the total fluctuation $g_{\epsilon}$.

2.3.2. Control on the relaxation. The second use of the scaled relative entropy inequality is to control the relaxation.

Rewriting the kinetic equation in terms of the fluctuation, we get

$$
\epsilon \partial_{t} g_{\epsilon}+v \cdot \nabla_{x} g_{\epsilon}=-\frac{1}{\epsilon} \mathcal{L} g_{\epsilon}+\mathcal{Q}\left(g_{\epsilon}, g_{\epsilon}\right) .
$$

We deduce formally that $g_{\epsilon}$ should be close to its orthogonal projection on Ker $\mathcal{L}$, which is the expected relaxation toward the infinitesimal Maxwellian.

The previous argument, however, is not correct in the framework of renormalized solutions, since

- the kinetic equation is not known to be satisfied (even in the sense of distributions);

- the fluctuation $g_{\epsilon}$ is not in $L^{2}$, so that the Hilbertian theory of $\mathcal{L}$ cannot be applied.

Our starting point here is therefore the following identity for the renormalized fluctuation,

$$
\frac{1}{\epsilon} \mathcal{L} \hat{g}_{\epsilon}=\frac{1}{4} \mathcal{Q}\left(\hat{g}_{\epsilon}, \hat{g}_{\epsilon}\right)-\frac{1}{\epsilon^{2}} \frac{1}{M} Q\left(\sqrt{M f_{\epsilon}}, \sqrt{M f_{\epsilon}}\right),
$$

based on the bilinearity of $Q$.

The estimate on the renormalized fluctuation obtained in the previous paragraph, together with easy continuity properties of $Q$ (possibly after suitable truncations of the collision cross-section $b$ ), provides a control on the first term in (2.9):

$$
\mathcal{Q}\left(\hat{g}_{\epsilon}, \hat{g}_{\epsilon}\right)=O(1)_{L^{1}\left(d x, L^{2}(M d v)\right)} \text {. }
$$

The second term in the right-hand side of (2.9), which is a kind of renormalized collision term, is controlled by the entropy dissipation

$$
\left\|\frac{1}{\epsilon^{2}} Q\left(\sqrt{M f_{\epsilon}}, \sqrt{M f_{\epsilon}}\right)\right\|_{L^{2}(M d v)}^{2} \leq \frac{4}{\epsilon^{4}} D\left(f_{\epsilon}\right),
$$

using the same kind of functional inequality as for (2.7).

We therefore end up with the relaxation estimate

$$
\hat{g}_{\epsilon}-\left(\hat{\rho}_{\epsilon}+\hat{u}_{\epsilon} \cdot v+\hat{\theta}_{\epsilon} \frac{|v|^{2}-3}{2}\right)=O(\epsilon)_{L_{t}^{2}\left(L_{x}^{1}\left(L^{2}(M d v)\right)\right.},
$$

denoting by $\hat{\rho}_{\epsilon}, \hat{u}_{\epsilon}$, and $\hat{\theta}_{\epsilon}$ the density, bulk velocity, and temperature, respectively, associated to the fluctuation $\hat{g}_{\epsilon}$.

Remark 2.4. Of course this step is specific to the study of hydrodynamic limits of the Boltzmann equation, and has no analogy at the level of the incompressible Navier-Stokes equations: it corresponds indeed to the control of the purely kinetic part of the solution, the one which is precisely neglected in the fluid approximation.

This estimate is however crucial in order to understand the dissipation process, which also appears at the macroscopic level and will be studied in the next section. 


\section{Some mathematical tools for the analysis of CONVERGence}

Since we just want to describe the limiting fluctuation $g$, the only remaining point is to establish that its moments $\rho, u$, and $\theta$ satisfy the incompressible Navier-Stokes equations. This program, suggested by Grad in the framework of the compressible Euler asymptotics, has been developed by Bardos, Golse and Levermore [3] in the viscous inviscid regime. We will not give here all the details of the proof, instead we refer to [21, but insist on just the key arguments.

3.1. Renormalization and conservation defects. The first difficulty is related to the fact that the only solutions to the Boltzmann equation which are known to exist globally in time (without any restriction on the size or the regularity of the initial data) are renormalized solutions.

Because of the symmetry breaking, renormalized solutions are not known to satisfy conservation laws. More precisely, only the local conservation of mass and the global conservation of momentum are satisfied. Since these conservation laws are expected to provide the incompressible Navier-Stokes and Fourier equations as $\epsilon \rightarrow 0$, one has first to establish that the conservation defects vanish asymptotically.

To do so, we start with the following approximate conservation laws

$$
\partial_{t} \int M g_{\epsilon} \gamma_{\epsilon} \varphi_{\epsilon} d v+\frac{1}{\epsilon} \nabla_{x} \cdot \int M g_{\epsilon} \gamma_{\epsilon} \varphi_{\epsilon} v d v=\frac{1}{\epsilon^{3}} \int \hat{\gamma}_{\epsilon} Q\left(f_{\epsilon}, f_{\epsilon}\right) \varphi_{\epsilon} d v,
$$

where $\gamma_{\epsilon}=\gamma\left(1+\epsilon g_{\epsilon}\right), \hat{\gamma}_{\epsilon}=\epsilon g_{\epsilon} \gamma^{\prime}\left(1+\epsilon g_{\epsilon}\right)+\gamma\left(1+\epsilon g_{\epsilon}\right)$ and $\gamma$ is a truncation satisfying the conditions

$$
\gamma \in C_{c}^{\infty}\left(\mathbf{R}^{+}\right), \quad \gamma(z) \equiv 1 \text { on }[0,2],
$$

and $\varphi_{\epsilon}$ is a truncated collision invariant

$$
\varphi_{\epsilon}=\varphi \mathbf{1}_{|v|^{2} \leq K_{\epsilon}} \quad \text { with } \varphi \in \operatorname{span}\left(1, v,|v|^{2}\right) .
$$

The conservation defects are then decomposed by introducing the renormalized collision integrand

$$
q_{\epsilon}=\frac{1}{\epsilon^{2}}\left(\sqrt{f_{\epsilon}^{\prime} f_{\epsilon 1}^{\prime}}-\sqrt{f_{\epsilon} f_{\epsilon 1}}\right)
$$

which is controlled by the entropy dissipation. More precisely,

$$
\begin{aligned}
D_{\epsilon}(\varphi) \equiv & \frac{1}{\epsilon^{3}} \int \hat{\gamma}_{\epsilon} Q\left(f_{\epsilon}, f_{\epsilon}\right) \varphi_{\epsilon} d v \\
=\epsilon & \iiint \hat{\gamma}_{\epsilon} \mathbf{1}_{|v|^{2} \leq K_{\epsilon}} q_{\epsilon}^{2} \varphi b d v d v_{1} d \sigma \\
& -\frac{2}{\epsilon} \iiint \hat{\gamma}_{\epsilon}\left(1-\mathbf{1}_{|v|^{2} \leq K_{\epsilon}}\right) q_{\epsilon} \varphi b d v d v_{1} d \sigma \\
& +\frac{2}{\epsilon} \iiint \hat{\gamma}_{\epsilon}\left(1-\hat{\gamma}_{\epsilon 1}\right) q_{\epsilon} \sqrt{f_{\epsilon} f_{\epsilon 1}} \varphi b d v d v_{1} d \sigma \\
& +\frac{2}{\epsilon} \iiint \hat{\gamma}_{\epsilon} \hat{\gamma}_{\epsilon 1}\left(1-\hat{\gamma}_{\epsilon}^{\prime} \hat{\gamma}_{\epsilon 1}^{\prime}\right) q_{\epsilon} \sqrt{f_{\epsilon} f_{\epsilon 1}} \varphi b d v d v_{1} d \sigma \\
& -\frac{\epsilon}{2} \iiint \hat{\gamma}_{\epsilon} \hat{\gamma}_{\epsilon}^{\prime} \hat{\gamma}_{\epsilon 1} \hat{\gamma}_{\epsilon 1}^{\prime} q_{\epsilon}^{2}\left(\varphi+\varphi_{1}\right) b d v d v_{1} d \sigma
\end{aligned}
$$

where we have used that $\varphi$ is a collision invariant to symmetrize the last term. 
The different terms are then estimated using:

- the entropy dissipation bound

$$
\int_{0}^{t} d s \int d x \iint q_{\epsilon}^{2} b d v d v_{1} d \sigma \leq C_{0}
$$

- Gaussian tails

$$
\int_{|v|^{2}>R}|v|^{p} M(v) d v \sim \sqrt{\frac{2}{\pi}} R^{(p+1) / 2} e^{-R / 2} ;
$$

- and the convergence

$$
\frac{1-\gamma_{\epsilon}}{\epsilon} \rightarrow 0 \text { in } L_{\mathrm{loc}}^{2}\left(d t d x, L^{p}(M d v)\right),
$$

which results from the equi-integrability

$$
\left|\hat{g}_{\epsilon}\right|^{2} \text { is weakly compact in } L_{\text {loc }}^{1}\left(d t d x, L^{1}(M d v)\right) \text {. }
$$

Such an integrability (or equivalently a $L^{1}$-weak compactness) is not a consequence of the entropy and entropy dissipation bounds. For instance, it is not known to hold in inviscid regimes (when the Knudsen number is very small compared to the Mach number). At this stage, (3.3) is therefore an assumption that we will have to check later on.

3.2. Hypoellipticity. Controlling the spatial regularity of the fluctuation (or at least of its moments) is the second major challenge of the proof. The main idea is that the entropy dissipation controls the regularity with respect to velocity variables, and that this regularity is then transferred to spatial variables by hypoellipticity. The entropy dissipation is therefore used twice, both

- to control the regularity with respect to $v$ and

- to control the source terms of the free transport.

Let us sketch briefly the main steps to obtain these two types of estimates.

3.2.1. Weak compactness in $v$. The equi-integrability with respect to $v$ comes from the relaxation and, more precisely, from the decomposition

$$
\hat{g}_{\epsilon}=\Pi \hat{g}_{\epsilon}+\left(\hat{g}_{\epsilon}-\Pi \hat{g}_{\epsilon}\right) \text {, }
$$

which is the linear version of the first-order Chapman-Enskog expansion.

The first term is obviously smooth in $v$ since, by definition,

$$
\Pi \hat{g}_{\epsilon}=\hat{\rho}_{\epsilon}+\hat{u}_{\epsilon} \cdot v+\hat{\theta}_{\epsilon} \frac{|v|^{2}-3}{2},
$$

where $\left(\hat{\rho}_{\epsilon}, \hat{u}_{\epsilon}, \hat{\theta}_{\epsilon}\right)$ are bounded in $L_{t}^{\infty}\left(L_{x}^{2}\right)$.

The second term is controlled by the entropy dissipation as shown in (2.9). We indeed have

$$
\left\|\hat{g}_{\epsilon}-\Pi \hat{g}_{\epsilon}\right\|_{L^{2}(M d v)}=O(\epsilon)_{L_{t}^{\infty}\left(L_{x}^{1}\right)}+O(\epsilon)_{L_{t, x}^{2}} .
$$

Combining this estimate with some pointwise bound, we find that for any $\lambda>0$

$$
M\left|\hat{g}_{\epsilon}\right|^{2} \mathbf{1}_{f_{\epsilon} \leq \lambda M} \text { is equi-integrable in } v .
$$

Note that large tails of the distribution $M\left|\hat{g}_{\epsilon}\right|^{2} \mathbf{1}_{f_{\epsilon}>\lambda M}$ as well as large velocities $M\left|\hat{g}_{\epsilon}\right|^{2}|v|^{2}$ can be controlled directly by the relative entropy (see [39] for instance), so that we end up with the following estimate: for any $p<2$,

$$
M\left|\hat{g}_{\epsilon}\right|^{2}\left(1+|v|^{p}\right) \text { is equi-integrable in } v .
$$


3.2.2. Regularizing properties of the free transport operator. The core of the proof is then to obtain the equi-integrability of $M \hat{g}_{\epsilon}^{2}$ with respect to spatial variables (see (3.3) ) and the strong compactness of the moments $\hat{\rho}_{\epsilon}, \hat{u}_{\epsilon}$, and $\hat{\theta}_{\epsilon}$. Both properties come from the structure of the free transport operator and, more precisely, from hypoellipticity and averaging lemma.

Hypoellipticity allows to transfer regularity or integrability from the $v$ variable to the $x$ variable. The result we need here is the following local dispersion statement [22]:

Theorem 3.1 (Golse and Saint-Raymond). Let $\left(\beta_{\epsilon}\right)$ be a bounded family in $L_{t}^{\infty}\left(L_{x, v}^{1}\right)$ such that

$$
\begin{gathered}
\left(\epsilon \partial_{t} \beta_{\epsilon}+v \cdot \nabla_{x} \beta_{\epsilon}\right) \text { is bounded in } L_{t, x, v}^{1}, \\
\left(\beta_{\epsilon}\right) \text { is uniformly equi-integrable in } v .
\end{gathered}
$$

Then the family $\left(\beta_{\epsilon}\right)$ is uniformly equi-integrable (in all variables) and thus weakly compact by the Dunford-Pettis criterion.

Note that extensions of this statement (required, for instance, when considering additional force terms in the transport equation [2]) can be obtained by using refined arguments of harmonic analysis based on a careful splitting of the phase space according to the symbol of the free transport operator. We refer to [1] for more precise statements.

The averaging lemma, already stated in Theorem 1.2, exploits another feature of the free transport operator, namely the fact that outside of a small subset of the phase space, it behaves as an elliptic operator.

It has been improved in many directions, especially in [16] to account for derivatives in $v$ in the source term, but we will not need these refinements here.

3.2.3. Control of the source terms. The key point is therefore to obtain a control on the transport, which is done essentially by the same techniques as for the conservation defects, except that we neither use the symmetries of the collision integrand nor the equi-integrability (3.3). What can be proved is the following estimate:

$$
\left(\epsilon \partial_{t}+v \cdot \nabla_{x}\right) M \hat{g}_{\epsilon, a}=O\left(\epsilon^{2-a / 2}\right)_{L_{t, x, v}^{1}}+O(1)_{L_{t, x, v}^{2}}+O(\epsilon)_{L_{\mathrm{loc}}^{1}\left(d t d x, L_{v}^{2}\right)},
$$

where

$$
\hat{g}_{\epsilon, a}=\frac{2}{\epsilon}\left(\sqrt{f_{\epsilon} / M+\epsilon^{a}}-1\right) .
$$

At this stage using both Theorems 1.2 and 3.1 we have therefore the strong compactness with respect to $x$ of the moments $\left(\hat{\rho}_{\epsilon}, \hat{u}_{\epsilon}, \hat{\theta}_{\epsilon}\right)$ and the fact that they satisfy some approximate conservation laws.

3.3. Compensated compactness. The last step is to take limits in these approximate conservation laws. Using the identity (2.9),

$$
\frac{1}{\epsilon} \mathcal{L} \hat{g}_{\epsilon}=\frac{1}{4} \mathcal{Q}\left(\hat{g}_{\epsilon}, \hat{g}_{\epsilon}\right)-\iint q_{\epsilon} \sqrt{M_{1} M} b d v d v_{1} d \omega
$$

together with the fact that $\left(\hat{g}_{\epsilon}, q_{\epsilon}\right)$ satisfies

$$
\left(v \cdot \nabla_{x}\right) M \hat{g}_{\epsilon}=\iint q_{\epsilon} \sqrt{M M_{1}} b d v d v_{1} d \omega+o(1),
$$


we decompose the momentum flux (resp. the energy flux) as the sum of a nonlinear convection term and a diffusion term. Denoting by $\mathbf{P}$ the Leray projection, we have

$\partial_{t} \mathbf{P} u_{\epsilon}+\mathbf{P} \nabla_{x} \cdot\left(u_{\epsilon} \otimes u_{\epsilon}\right)-\mu \Delta_{x} \mathbf{P} u_{\epsilon} \rightarrow 0$ in the sense of distributions,

$\partial_{t} \frac{1}{5}\left(3 \theta_{\epsilon}-2 \rho_{\epsilon}\right)+\nabla_{x} \cdot\left(\theta_{\epsilon} u_{\epsilon}\right)-\kappa \Delta_{x} \theta_{\epsilon} \rightarrow 0$ in the sense of distributions.

The difficulty is therefore to obtain the weak stability of the convection terms. We indeed know that $\left(u_{\epsilon}\right)$ is strongly compact with respect to $x$, but we do not expect any uniform regularity with respect to time.

More precisely, because the incompressibility and Boussinesq constraints are not satisfied for fixed $\epsilon$, fast temporal oscillations (referred to as acoustic waves) arise in the system. Integrating the transport equation (3.1) and using the ChapmanEnskog decomposition (3.4), we actually find that

$$
\begin{aligned}
\epsilon \partial_{t} \rho_{\epsilon}+\nabla_{x} \cdot u_{\epsilon} & =O(\epsilon)_{L_{\mathrm{loc}}^{1}\left(d t, W_{\mathrm{loc}}^{-1,1}(d x)\right)}, \\
\epsilon \partial_{t} u_{\epsilon}+\nabla_{x}\left(\rho_{\epsilon}+\theta_{\epsilon}\right) & =O(\epsilon)_{L_{\mathrm{loc}}^{1}\left(d t, W_{\mathrm{loc}}^{-1,1}(d x)\right)}, \\
\epsilon \partial_{t}\left(3 \theta_{\epsilon}-2 \rho_{\epsilon}\right) & =O(\epsilon)_{L_{\mathrm{loc}}^{1}\left(d t, W_{\mathrm{loc}}^{-1,1}(d x)\right)}
\end{aligned}
$$

It is thus natural to decompose

$$
\begin{gathered}
u_{\epsilon}=\bar{u}_{\epsilon}+\nabla \psi_{\epsilon} \text { with } \bar{u}_{\epsilon}=\mathbf{P} u_{\epsilon}, \\
\rho_{\epsilon}=-\bar{\theta}_{\epsilon}+\pi_{\epsilon}, \quad \theta_{\epsilon}=\bar{\theta}_{\epsilon}+\frac{2}{3} \pi_{\epsilon} \text { with } \bar{\theta}_{\epsilon}=\frac{1}{5}\left(3 \theta_{\epsilon}-2 \rho_{\epsilon}\right) .
\end{gathered}
$$

We then expect $\bar{u}_{\epsilon}$ and $\bar{\theta}_{\epsilon}$ to be strongly compact both in $t$ and $x$ (which can be proved using some interpolation lemma together with a careful study of the Leray projection). The question is to understand how the fast oscillating components $\nabla \psi_{\epsilon}$ and $\pi_{\epsilon}$ interact and possibly create interferences preventing the convergence of the product

$$
\mathbf{P} \nabla_{x} \cdot\left(u_{\epsilon} \otimes u_{\epsilon}\right) \rightarrow \mathbf{P} \nabla_{x} \cdot(\bar{u} \otimes \bar{u}) .
$$

Here, compensated compactness will replace the usual weak-strong compactness; it relies both on the algebraic structure of the nonlinearity and on the spectral description of the wave operator. The key result, obtained by Lions and Masmoudi in [34], is the following

Theorem 3.2 (Lions and Masmoudi). Consider two families $\pi_{\epsilon}$ and $\nabla \psi_{\epsilon}$ uniformly bounded in $L_{t}^{\infty}\left(L_{x}^{2}\right)$ and strongly compact in $x$. Assume that

$$
\begin{aligned}
\epsilon \partial_{t} \pi_{\epsilon}+\Delta_{x} \psi_{\epsilon} & =o(1)_{L_{t}^{1}\left(W_{x}^{-s, 1}\right)}, \\
\epsilon \partial_{t} \nabla \psi_{\epsilon}+\frac{5}{3} \nabla_{x} \pi_{\epsilon} & =o(1)_{L_{t}^{1}\left(W_{x}^{-s, 1}\right)},
\end{aligned}
$$

for some $s>1$. Then,

$$
\mathbf{P} \nabla_{x} \cdot\left(\left(\nabla \psi_{\epsilon}\right)^{\otimes 2}\right) \rightarrow 0 \quad \text { and } \nabla_{x} \cdot\left(\pi_{\epsilon} \nabla \psi_{\epsilon}\right) \rightarrow 0
$$

in the sense of distributions. 
For the sake of completeness, we recall here the formal computation leading to these statements:

$$
\begin{aligned}
\nabla_{x} \cdot\left(\left(\nabla \psi_{\epsilon}\right)^{\otimes 2}\right) & =\frac{1}{2} \nabla_{x}\left(\left|\nabla_{x} \psi_{\epsilon}\right|^{2}\right)+\nabla_{x} \psi_{\epsilon} \Delta_{x} \psi_{\epsilon}=\frac{1}{2} \nabla_{x}\left(\left|\nabla_{x} \psi_{\epsilon}\right|^{2}\right)+\nabla_{x} \psi_{\epsilon}\left(s_{\epsilon}^{0}-\epsilon \partial_{t} \pi_{\epsilon}\right) \\
& =\frac{1}{2} \nabla_{x}\left(\left|\nabla_{x} \psi_{\epsilon}\right|^{2}\right)+\nabla_{x} \psi_{\epsilon} s_{\epsilon}^{0}-\epsilon \partial_{t}\left(\pi_{\epsilon} \nabla_{x} \psi_{\epsilon}\right)+\pi_{\epsilon}\left(s_{\epsilon}-\frac{5}{3} \nabla_{x} \pi_{\epsilon}\right) \\
& =\frac{1}{2} \nabla_{x}\left(\left|\nabla_{x} \psi_{\epsilon}\right|^{2}-\frac{5}{3}\left(\pi_{\epsilon}\right)^{2}\right)-\epsilon \partial_{t}\left(\pi_{\epsilon} \nabla_{x} \psi_{\epsilon}\right)+\left(\nabla_{x} \psi_{\epsilon}\right) s_{\epsilon}^{0}+\pi_{\epsilon} s_{\epsilon},
\end{aligned}
$$

denoting $s_{\epsilon}^{0}=\epsilon \partial_{t} \pi_{\epsilon}+\Delta_{x} \psi_{\epsilon}$ and $s_{\epsilon}=\epsilon \partial_{t} \nabla \psi_{\epsilon}+\frac{5}{3} \nabla_{x} \pi_{\epsilon}$. An analogous identity holds for the energy transport

$$
\begin{aligned}
\nabla_{x} \cdot\left(\pi_{\epsilon} \nabla_{x} \psi_{\epsilon}\right) & =\pi_{\epsilon}^{\delta} \Delta_{x} \psi_{\epsilon}+\nabla_{x} \pi_{\epsilon} \cdot \nabla_{x} \psi_{\epsilon} \\
& =\pi_{\epsilon}\left(s_{\epsilon}^{0}-\epsilon \partial_{t} \pi_{\epsilon}\right)+\nabla_{x} \psi_{\epsilon} \cdot \frac{3}{5}\left(s_{\epsilon}-\epsilon \partial_{t} \nabla_{x} \psi_{\epsilon}\right) \\
& =-\frac{\epsilon}{2} \partial_{t}\left(\left(\pi_{\epsilon}\right)^{2}+\frac{3}{5}\left|\nabla \psi_{\epsilon}\right|^{2}\right)+\pi_{\epsilon} s_{\epsilon}^{0}+\frac{3}{5} \nabla_{x} \psi_{\epsilon} \cdot s_{\epsilon} .
\end{aligned}
$$

The rigorous justification of these computations would require a regularization with respect to spatial variables, which will not be detailed in this review paper. We only note that the spatial regularity is needed here.

We finally end up with the following convergence result, established in [20] for Maxwellian cross-sections and in 21] for general hard potentials with cut-off:

Theorem 3.3 (Golse and Saint-Raymond). Let $\left(f_{\epsilon}^{i n}\right)$ be a family of initial data such that

$$
\frac{1}{\epsilon^{2}} H\left(f_{\epsilon}^{i n} \mid M\right) \leq C_{0}
$$

For any fixed $\epsilon$, let $f_{\epsilon}=M\left(1+\epsilon g_{\epsilon}\right)$ be a renormalized solution to the scaled Boltzmann equation (2.1). Then $\left(g_{\epsilon}\right)$ is relatively weakly compact in $L_{\mathrm{loc}}^{1}(d t d x M d v)$, and any limit point $g$ of $\left(g_{\epsilon}\right)$ satisfies

$$
g=\Pi g=\rho+u \cdot v+\theta \frac{|v|^{2}-3}{2},
$$

where

$$
\begin{array}{r}
\partial_{t} u+u \cdot \nabla_{x} u+\nabla p-\mu \Delta_{x} u=0, \quad \nabla_{x} \cdot u=0, \\
\partial_{t} \theta+u \cdot \nabla_{x} \theta-\kappa \Delta_{x} \theta=0, \quad \nabla_{x}(\rho+\theta)=0 .
\end{array}
$$

This actually justifies the Chapman-Enskog expansion up to second order

$$
\begin{array}{r}
\Pi \hat{g}_{\epsilon}=\hat{\rho}_{\epsilon}+\hat{u}_{\epsilon} \cdot v+\hat{\theta}_{\epsilon} \frac{|v|^{2}-3}{2}, \\
\hat{g}_{\epsilon}-\Pi \hat{g}_{\epsilon}=O(\epsilon),
\end{array}
$$

where $\left(\hat{\rho}_{\epsilon}, \hat{u}_{\epsilon}, \hat{\theta}_{\epsilon}\right)$ are approximate solutions to the Navier-Stokes Fourier equations. But this does not tell us anything about the size of the error made by replacing $\left(\hat{\rho}_{\epsilon}, \hat{u}_{\epsilon}, \hat{\theta}_{\epsilon}\right)$ by the solutions to the Navier-Stokes Fourier equations.

\section{WEAK CONVERGENCE VERSUS STRONG CONVERGENCE}

Additional information on the asymptotics may be obtained by considering strong convergence rather than weak convergence. Note that this will not give higher approximations of the Chapman-Enskog expansion but only a more precise 
description of $O(1)$ terms: we indeed expect to catch oscillations and concentrations if they exist in the system.

The strategy of the proof is then based on some weak-strong stability principle, meaning that we will compare the solution of the scaled Boltzmann equation to its formal asymptotics in some suitable "distance", here by computing their relative entropy. The idea of using the relative entropy for the study of hydrodynamic limits goes back to Yau [43. It has been first implemented for the Boltzmann equation in 19, then improved by the author 37. to get some convergence result in the incompressible Euler limit under the only assumption that the initial data is well prepared.

Dealing with ill-prepared data is more difficult since we have to describe both the acoustic waves and the initial layer (assuming for the sake of simplicity that there is either no spatial boundary or purely specular reflection at the boundary). A first attempt to extend the relative entropy method in this case has been made in the incompressible inviscid setting [38, but it requires additional assumptions on the renormalized solutions in order that the conservation laws are satisfied and that the energy flux can be controlled.

In viscous regimes, such assumptions are not compulsory insofar as one can introduce approximate conservation laws and prove that the conservation defects vanish asymptotically. The idea is to modulate not the entropy itself, but a functional which is controlled by the entropy. This functional is essentially the $L^{2}$-norm of the renormalization $g_{\epsilon} \gamma_{\epsilon}$; we therefore work in a Hilbertian setting and with approximate conservation laws.

This remark has been developed in recent work with D. Arsenio 2] and is fundamental when dealing with magneto-hydrodynamic limits of the Vlasov-MaxwellBoltzmann system leading to Ohm's law, since conservation defects can be controlled but weak stability is not known to hold in this kind of regime.

Define the modulated renormalized entropy by

$$
\delta \mathcal{H}(t)=\frac{1}{2} \iint M\left(g_{\epsilon} \gamma_{\epsilon} \mathbf{1}_{|v|^{2} \leq K_{\epsilon}}-\left(\rho+u \cdot v+\theta \frac{|v|^{2}-3}{2}\right)\right)^{2} d v d x
$$

for some renormalization such that

$$
\gamma(z) \equiv 1 \text { in the vicinity of } 1, \quad \gamma(z) \sim \sqrt{z} \text { at infinity, }
$$

and the modulated renormalized entropy dissipation by

$$
\delta \mathcal{D}(t)=\frac{1}{4} \iint\left(q_{\epsilon}-q\right)^{2} b M M_{1} d v d v_{1} d \omega d x,
$$

where

$$
q=\frac{1}{4} \nabla_{x} u:\left(\tilde{\Phi}+\tilde{\Phi}_{1}-\tilde{\Phi}^{\prime}-\tilde{\Phi}_{1}^{\prime}\right)+\frac{1}{4} \nabla_{x} \theta \cdot\left(\tilde{\Psi}+\tilde{\Psi}_{1}-\tilde{\Psi}^{\prime}-\tilde{\Psi}_{1}^{\prime}\right)
$$

denoting by $\Phi$ and $\Psi$ the kinetic momentum and energy fluxes

$$
\Phi=v^{\otimes 2}-\frac{1}{3}|v|^{2} I d, \quad \Psi=\frac{1}{2} v\left(|v|^{2}-5\right),
$$

and by $\tilde{\Phi}$ and $\tilde{\Psi}$ their respective images by $\mathcal{L}^{-1}$. 
What can be proved is the stability inequality

$$
\begin{aligned}
& \delta \mathcal{H}(t)+\int_{0}^{t} \mathcal{D}(s) d s \\
& \leq \frac{1}{\epsilon^{2}} H\left(f_{\epsilon}^{i n} \mid M_{\exp \left(\epsilon \rho^{i n}, \epsilon u^{i n}, \exp \left(\epsilon \theta^{i n}\right) \exp (\gamma(t))\right.}\right. \\
& -\int_{0}^{t} \int\left(\begin{array}{c}
\rho_{\epsilon}-\rho \\
u_{\epsilon}-u \\
\theta_{\epsilon}-\theta
\end{array}\right) \cdot \mathbf{A}_{\epsilon}(\rho, u, \theta) \exp (\gamma(s)) d x d s+o(1),
\end{aligned}
$$

where the acceleration operator is defined by

$$
\mathbf{A}_{\epsilon}(\rho, u, \theta)=\left(\begin{array}{c}
\partial_{t} \rho+\frac{1}{\epsilon} \nabla_{x} \cdot u+u \cdot \nabla_{x} \rho \\
\partial_{t} u+\frac{1}{\epsilon} \nabla_{x}(\rho+\theta)+\theta \nabla_{x}\left(\rho-\frac{3}{2} \theta\right)+u \cdot \nabla_{x} u-\mu \Delta_{x} u \\
\partial_{t} \theta+\frac{2}{3 \epsilon} \nabla_{x} \cdot u+u \cdot \nabla_{x} \theta-\kappa \Delta_{x} \theta
\end{array}\right)
$$

and the growth rate is given by

$$
\gamma(t)=\int_{0}^{t}\left(\|u(s)\|_{W^{1, \infty}}+\|\theta(s)\|_{W^{1, \infty}}\right) d s .
$$

The main ingredients to prove this stability inequality are the scaled entropy inequality (2.4) and the approximate conservation laws obtained by (3.1) together with the convergence of conservation defects. Actually, in order to obtain a rate of convergence, we do not exactly use the convergence to 0 of conservation defects based on the equi-integrability statement (3.3) (which is not quantitative) but rather a control of conservation defects by the modulated entropy and some Gronwall inequality. For the precise computations of the renormalized relative entropy and the proof of stability, we refer to [2].

We will focus here on the construction of the approximate solution as the sum of different contributions, the main one being

$$
g=\bar{\rho}+\bar{u} \cdot v+\bar{\theta} \frac{|v|^{2}-3}{2},
$$

where $(\bar{\rho}, \bar{u}, \bar{\theta})$ satisfy the Navier-Stokes-Fourier system (3.7).

4.1. Fast time oscillations. Acoustic waves only contribute to the hydrodynamic part of the distribution:

$$
g_{\mathrm{osc}}=\rho_{\mathrm{osc}}+u_{\mathrm{osc}} \cdot v+\theta_{\mathrm{osc}} \frac{|v|^{2}-3}{2},
$$

where $\left(\bar{\rho}+\rho_{\text {osc }}, \bar{u}+u_{\text {osc }}, \bar{\theta}+\theta_{\text {osc }}\right)$ are approximate solutions to the singular perturbation problem

$$
\mathbf{A}_{\epsilon}(\rho, u, \theta)=0 .
$$

More precisely, we will require that

$$
\mathbf{A}_{\epsilon}\left(\bar{\rho}+\rho_{\mathrm{osc}}, \bar{u}+u_{\mathrm{osc}}, \bar{\theta}+\theta_{\mathrm{osc}}\right) \rightarrow 0 \text { in } L^{2}(d t d x) \text { as } \epsilon \rightarrow 0 .
$$

Such a construction is done by a filtering method (see 27] or 40, for instance), i.e., considering the family $\mathcal{W}\left(\frac{t}{\epsilon}\right)(\rho, u, \theta)$, where $\mathcal{W}$ is the semigroup generated by 
the linear penalization

$$
W(\rho, u, \theta)=\left(\nabla_{x} \cdot u, \nabla_{x}(\rho+\theta), \frac{2}{3} \nabla_{x} \cdot u\right) .
$$

The first-order approximation is then obtained by taking (strong) limits in the filtered system. Nevertheless, because of the high frequency oscillations, we do not expect the error in this first-order approximation to converge strongly to 0 .

We therefore have to add some correctors (i.e., the second-order approximation) in order to establish the convergence statement (4.2).

More precisely, we have the following

Proposition 4.1. Let $\left(\rho_{i n}, u_{i n}, \theta_{i n}\right)$ belong to $H^{s}(\Omega)$ for some $s>\frac{5}{2}$. Then there exist some $t^{*}>0$ and some family $\left(\rho_{\mathrm{osc}}^{N}, u_{\mathrm{osc}}^{N}, \theta_{\mathrm{osc}}^{N}\right)$ satisfying the uniform bound

$$
\sup _{N \in \mathbf{N}} \lim _{\epsilon \rightarrow 0}\left\|\left(\rho_{\mathrm{osc}}^{N}, u_{\mathrm{osc}}^{N}, \theta_{\mathrm{osc}}^{N}\right)\right\|_{L^{1}\left(\left[0, t^{*}\right], H^{s}(\Omega)\right)} \leq C
$$

and such that the following convergences hold as $\epsilon \rightarrow 0$ then $N \rightarrow \infty$ :

$$
\begin{gathered}
\left.\left(\bar{\rho}+\rho_{\mathrm{osc}}^{N}, \bar{u}+u_{\mathrm{osc}}^{N}, \bar{\theta}+\theta_{\mathrm{osc}}^{N}\right)\right)_{\mid t=0} \rightarrow\left(\rho_{\text {in }}, u_{i n}, \theta_{\text {in }}\right) \text { in } H^{s}(d x), \\
\mathbf{A}_{\epsilon}\left(\bar{\rho}+\rho_{\mathrm{osc}}^{N}, \bar{u}+u_{\mathrm{osc}}^{N}, \bar{\theta}+\theta_{\mathrm{osc}}^{N}\right) \rightarrow 0 \text { in } L^{2}(d t d x) .
\end{gathered}
$$

Note that, because of the structure of the nonlinearity already exhibited in section 3.3, the filtered system can be decoupled at leading order in

- an independent system for the projection $(\bar{\rho}, \bar{u}, \bar{\theta})$ on the kernel of $W$ and

- a linear transport-diffusion equation (with coefficients depending on $(\bar{\rho}, \bar{u}, \bar{\theta}))$ for the oscillating part $\mathcal{W}\left(\frac{t}{\epsilon}\right)\left(\rho_{\text {osc }}, u_{\text {osc }}, \theta_{\text {osc }}\right)$.

This structure is crucial for establishing the existence of smooth solutions and then for constructing the correctors by linearization techniques.

4.2. Relaxation layers. For general initial data, the purely kinetic part of the solution to the Boltzmann equation is expected to converge to 0 exponentially in time, in particular in $L_{\mathrm{loc}}^{1}(d t d x d v)$, but not in $L_{\mathrm{loc}}^{\infty}\left(d t, L_{\mathrm{loc}}^{1}(d x d v)\right)$. We have therefore to introduce another corrector, which is essentially localized in a thin time interval of size $\epsilon^{2}$.

In this initial layer, the dominant process is expected to be the relaxation, so that the transport can be neglected in first approximation. Nonlinear effects are also small compared to the linear relaxation. We thus solve the homogeneous linear equation

$$
\begin{array}{r}
\partial_{t} g_{\text {rel }}=\frac{1}{\epsilon^{2}} \mathcal{L} g_{\text {rel }}, \\
g_{\text {rel } \mid t=0}=g_{\text {in }} .
\end{array}
$$

The coercivity estimate for $\mathcal{L}$ ensures that $g_{\text {rel }}$ tends exponentially in $\left(t / \epsilon^{2}\right)$ to the infinitesimal Maxwellian $\rho_{i n}+u_{i n} \cdot v+\theta_{i n} \frac{|v|^{2}-3}{2}$. Furthermore, the regularity with respect to $x$ is obviously propagated.

The point is then to prove that this approximation is relevant, in the sense that

$$
\frac{1}{2} \iint M\left(g_{\epsilon} \gamma_{\epsilon}-g_{\mathrm{rel}}\right)^{2} d x d v \rightarrow 0
$$


in the initial layer. This step is extremely technical in [38, insofar as:

- we used the nonlinear relaxation equation to build the approximation;

- we needed to truncate large velocities to ensure a suitable decay at infinity;

- we modulated the entropy and the entropy dissipation (which is not expected to converge to 0 in the initial layer), which are convex functionals but not associated to some Hilbertian structure.

Here the proof is much simpler. Once again we will not give all the details but just mention how to modulate the dissipation.

Define the modulated renormalized entropy by

$$
\delta \mathcal{H}(t)=\frac{1}{2} \iint M\left(g_{\epsilon} \gamma_{\epsilon} \mathbf{1}_{|v|^{2} \leq K_{\epsilon}}-g_{\text {rel }}\right)^{2} d v d x
$$

and the modulated renormalized entropy dissipation by

$$
\delta \mathcal{D}(t)=\frac{1}{4} \iint\left(q_{\epsilon}-q_{\mathrm{rel}}\right)^{2} b M M_{1} d v d v_{1} d \omega d x
$$

where

$$
q_{\mathrm{rel}}=\frac{1}{\epsilon}\left(g_{\mathrm{rel}}+g_{\mathrm{rel}, 1}-g_{\mathrm{rel}}^{\prime}-g_{\mathrm{rel}, 1}^{\prime}\right)
$$

What can be proved is the stability inequality (provided that $g \in L_{t, x, v}^{\infty}$ )

$$
\begin{aligned}
& \delta \mathcal{H}(t)+\int_{0}^{t} \mathcal{D}(s) d s \\
& \leq \frac{1}{\epsilon^{2}} H\left(f_{\epsilon}^{i n} \mid M\left(1+\epsilon g^{i n}\right)\right) \exp (\gamma(t)) \\
& -\int_{0}^{t} \int M\left(g_{\epsilon} \gamma_{\epsilon}-g_{\mathrm{rel}}\right)\left(\frac{1}{\epsilon} v \cdot \nabla_{x} g_{\mathrm{rel}}\right) \exp (\gamma(t)-\gamma(s)) d x d s+O\left(\frac{t}{\epsilon}\right) .
\end{aligned}
$$

The main difference with (4.1) comes from the facts that the transport is dealt with as a perturbation and that $g_{\text {rel }}$ is not a collision invariant, which introduces two additional terms. The first one comes from the time derivative of $g_{\text {rel }}$

$$
\begin{aligned}
-\frac{1}{\epsilon^{2}} & \int \mathcal{L} g_{\mathrm{rel}}\left(g_{\mathrm{rel}}-g_{\epsilon} \gamma_{\epsilon} \mathbf{1}_{|v|^{2} \leq K_{\epsilon}}\right) M d v \\
& =-\frac{1}{\epsilon^{2}} \int g_{\mathrm{rel}} \mathcal{L}\left(g_{\mathrm{rel}}-g_{\epsilon} \gamma_{\epsilon} \mathbf{1}_{|v|^{2} \leq K_{\epsilon}}\right) M d v \\
& =-\frac{1}{4} \iint q_{\mathrm{rel}}\left(q_{\mathrm{rel}}-q_{\epsilon}\right) b M M_{1} d v_{1} d v d \omega+O\left(\frac{1}{\epsilon}\right)_{L_{t}^{\infty}\left(L_{x}^{1}\right)},
\end{aligned}
$$

where we have used identity (2.9) to compute $\mathcal{L} \hat{g}_{\epsilon}$. 
The second one comes from the time derivative of $g_{\epsilon} \gamma_{\epsilon}$

$$
\begin{aligned}
\frac{1}{\epsilon^{3}} \int \hat{\gamma}_{\epsilon} Q\left(f_{\epsilon}, f_{\epsilon}\right) g_{\mathrm{rel}} d v \\
=\epsilon \iiint \hat{\gamma}_{\epsilon} q_{\epsilon}^{2} \varphi g_{\mathrm{rel}} d v d v_{1} d \sigma \\
+\frac{2}{\epsilon} \iiint \hat{\gamma}_{\epsilon}\left(1-\hat{\gamma}_{\epsilon 1}\right) q_{\epsilon} \sqrt{f_{\epsilon} f_{\epsilon 1}} g_{\mathrm{rel}} b d v d v_{1} d \sigma \\
+\frac{2}{\epsilon} \iiint \hat{\gamma}_{\epsilon} \hat{\gamma}_{\epsilon 1}\left(1-\hat{\gamma}_{\epsilon}^{\prime} \hat{\gamma}_{\epsilon 1}^{\prime}\right) q_{\epsilon} \sqrt{f_{\epsilon} f_{\epsilon 1}} g_{\mathrm{rel}} b d v d v_{1} d \sigma \\
\quad-\frac{\epsilon}{4} \iiint \hat{\gamma}_{\epsilon} \hat{\gamma}_{\epsilon}^{\prime} \hat{\gamma}_{\epsilon 1} \hat{\gamma}_{\epsilon 1}^{\prime} q_{\epsilon}^{2}\left(g_{\mathrm{rel}}+g_{\mathrm{rel}, 1}\right) b d v d v_{1} d \sigma \\
\quad-\frac{1}{4} \iiint \sqrt{f_{\epsilon} f_{\epsilon, 1}} \hat{\gamma}_{\epsilon} \hat{\gamma}_{\epsilon}^{\prime} \hat{\gamma}_{\epsilon 1} \hat{\gamma}_{\epsilon 1}^{\prime} q_{\epsilon} \frac{1}{\epsilon}\left(g_{\mathrm{rel}}+g_{\mathrm{rel}, 1}-g_{\mathrm{rel}}^{\prime}+g_{\mathrm{rel}, 1}^{\prime}\right) b d v d v_{1} d \sigma .
\end{aligned}
$$

The last term contributes to the modulated renormalized entropy dissipation, while the others are dealt with as in the case of conservation defects.

The inequality (4.6) shows that the purely kinetic part is negligible outside from the initial layer, the size of which is typically $O\left(\epsilon^{2}\right)$. Note however that it is not possible to use (4.6) in the initial layer, then (4.1) after a time $t_{\epsilon}$ such that $\epsilon^{2} \ll t_{\epsilon} \ll$ $\epsilon$. We indeed have no information on the relative entropy at time $t_{\epsilon}$, but only on a renormalized functional. We therefore have to build one approximate solution $(g, q)$ by combining the two previous constructions and using the modulated renormalized entropy inequality only once.

We finally end up with the following convergence result, which is obtained as a combination of the results obtained in [39] in the inviscid regime and of the renormalized entropy method introduced in [2]:

Theorem 4.2. Let $\left(f_{\epsilon, \text { in }}\right)$ be a family of initial data converging entropically at scale $\epsilon$ to some smooth fluctuation $g_{\text {in }}$

$$
\frac{1}{\epsilon}\left(f_{\epsilon, i n}-M\right) \rightarrow M g \text { in } L_{\mathrm{loc}}^{1}(d x d v) \text { and } \frac{1}{\epsilon^{2}} H\left(f_{\epsilon, i n} \mid M\right) \rightarrow \frac{1}{2} \iint M\left(g_{i n}\right)^{2} d v d x .
$$

For any fixed $\epsilon$, let $f_{\epsilon}=M\left(1+\epsilon g_{\epsilon}\right)$ be a renormalized solution to the scaled Boltzmann equation (2.1).

Let $g_{\text {app }}$ be the approximation defined by

$$
g_{\text {app }}=\exp \left(-\frac{t}{\epsilon^{2}} \mathcal{L}\right) \Pi^{\perp} g_{\text {in }}+\left(\bar{\rho}+\rho_{\text {osc }}\right)+\left(\bar{u}+u_{\text {osc }}\right) \cdot v+\left(\bar{\theta}+\theta_{\text {osc }}\right) \frac{|v|^{2}-3}{2},
$$

where $(\bar{\rho}, \bar{u}, \bar{\theta})$ satisfy the Navier-Stokes Fourier system (3.7), ( $\left.\rho_{\mathrm{osc}}, u_{\mathrm{osc}}, \theta_{\mathrm{osc}}\right)$ are the acoustic waves (defined in Proposition 4.1), and $\Pi^{\perp} g_{\text {in }}$ is the purely kinetic part of the initial fluctuation. Then $g_{\epsilon}-g_{\text {app }}$ converges strongly to 0 in $L_{\text {loc }}^{1}\left(d t d x, L^{1}\left(M\left(1+|v|^{2}\right) d v\right)\right)$.

Note that we could obtain a rate of convergence by a careful study of all error terms.

4.3. Regularity and uniqueness. In modulated entropy (or energy) methods, the regularity of the limiting field is the key point in proving the stability (and also to build the correctors for ill-prepared initial data). In hyperbolic regimes, we typically need some Lipschitz regularity to estimate the flux term by some 
Gronwall inequality. In parabolic regimes, it should be possible to relax a little bit this regularity assumption by using the a priori regularity on the moments coming from the entropy dissipation estimate and averaging lemma.

4.3.1. Weak-strong uniqueness. This is the counterpart at the kinetic level of the weak-strong stability, which has been stated by Leray for the solutions to the Navier-Stokes equations. For any weak (Leray) solution $u$ and any strong (Lipschitz) solution $\bar{u}$ of the Navier-Stokes equations, the following inequality holds:

$$
\|u(t)-\bar{u}(t)\|_{L^{2}}^{2} \leq\left\|u_{i n}-\bar{u}_{i n}\right\|_{L^{2}}^{2} \exp \left(C \int_{0}^{t}\|\nabla \bar{u}(s)\|_{L^{\infty}} d s\right) .
$$

It can be refined as

$$
\|u(t)-\bar{u}(t)\|_{L^{2}}^{2} \leq\left\|u_{i n}-\bar{u}_{i n}\right\|_{L^{2}}^{2} \exp \left(\frac{C}{\mu} \int_{0}^{t}\|\nabla \bar{u}(s)\|_{\dot{H}^{1 / 2}}^{2} d s\right),
$$

using Sobolev's embeddings together with some trilinear estimate for the flux term $\left|\int((u-\bar{u}) \cdot \nabla) \bar{u} \cdot(u-\bar{u})(s, x) d x\right| \leq C\|(u-\bar{u})(s)\|_{L^{2}}\|\nabla \bar{u}(s)\|_{\dot{H}^{1 / 2}}\|\nabla(u-\bar{u})(s)\|_{L^{2}}$.

Note that this is essentially the only setting for which the uniqueness of Leray solutions is guaranteed. It is then natural that strong convergence requires this level of regularity (since strong convergence of approximate solutions implies more or less uniqueness of the limit).

4.3.2. Dissipative solutions. Actually, the stability inequality that is established is a little bit more general insofar as we do not require that the test function $\bar{u}$ be a solution to the Navier-Stokes equations. What can be proved is typically the inequality

$$
\begin{aligned}
\|u(t)-\bar{u}(t)\|_{L^{2}}^{2} \\
\leq\left\|u_{i n}-\bar{u}_{i n}\right\|_{L^{2}}^{2} \exp \left(C \int_{0}^{t}\|\nabla \bar{u}(s)\|_{L^{\infty}} d s\right) \\
\quad-\int_{0}^{t} \int(u-\bar{u}) \cdot\left(\partial_{t} \bar{u}+\bar{u} \cdot \nabla_{x} \bar{u}-\mu \Delta_{x} \bar{u}\right)(s) \\
\quad \times \exp \left(C \int_{s}^{t}\left\|\nabla \bar{u}\left(s^{\prime}\right)\right\|_{L^{\infty}} d s^{\prime}\right) d s
\end{aligned}
$$

for any Lipschitz divergence-free vector field $\bar{u}$.

Starting from this remark, Lions has introduced 33 a very weak notion of solutions, referred to as dissipative solutions, defined by the constraint that $\nabla_{x} \cdot u=0$, in which $u$ is weakly continuous with respect to time and satisfies the stability inequality (4.7). By definition, these solutions coincide with the smooth solutions of the Navier-Stokes equations as long as they exist, but they are not known to be Leray solutions to the Navier-Stokes equations, nor to satisfy any renormalized form of the equations.

This means that the Leray solutions cannot be obtained in general as asymptotics of the scaled Boltzmann equation by entropy methods. In that respect, the relative entropy method is more restrictive than the weak compactness method described previously. 


\section{Consequences And Perspectives}

Even looking at only the first two orders in the Chapman-Enskog expansion, we have seen that both regularity and truncation are important and still-unsolved issues.

5.1. About the small parameter in the Chapman-Enskog expansion. Regularity is typically required to control the stability. For the time being, the only regime for which we are able to get enough a priori regularity to prove a rigorous convergence result is the one leading to the incompressible Navier-Stokes equations, which is the first-order correction to constant solutions of the compressible Euler equations. A natural question is then to know whether we can find other solutions to the compressible Euler equations that describe the asymptotics of the Boltzmann equation.

We have to mention here that rarefaction waves and shock waves of small amplitudes have been proved (in [10] and [36], respectively) to be limits of some Boltzmann dynamics. However the question we address here is much more general: we are not interested only in proving that one can build particular families of solutions to the scaled Boltzmann equation which have good asymptotics, but also in characterizing all the possible limit points in the fast relaxation limit.

- If the solution to the Euler equations is not smooth, as mentioned by Slemrod [42, it is not even clear what is the good notion of solution to be studied.

The Chapman-Enskog expansion requires in order to be consistent that the spatial derivatives are $o\left(\frac{1}{\epsilon}\right)$. This means that the weakly viscous approximation may be wrong, in which case the entropy criterion is not necessarily a good tool for selecting admissible solutions to the Euler equations.

- If we restrict our attention to smooth solutions to the Euler equations (which is of course a serious limitation), a natural idea is to extend the relative entropy method and to use the regularity of the limiting field to control the stability.

However, this strategy fails because of the lack of control on large velocities, which is a problem arising only at the kinetic level (we have no counterpart at the fluid level, which could be used for loop estimates). More precisely, the energy flux contains a moment of order 3 with respect to $v$, which cannot be controlled either by the relative entropy or by the entropy dissipation.

The point is that the Chapman-Enskog expansion actually involves powers of the scaled transport operator $\epsilon\left(v \cdot \nabla_{x}\right)$. Controlling large velocities is therefore as important as controlling the regularity.

In other words, because of large velocities and high frequencies, it is not clear at the present time whether or not the Chapman-Enskog expansion is relevant at any order (even one or two) to describe the asymptotics of the Boltzmann equation in the fast relaxation limit, except in very particular situations.

\subsection{About concentrations and large velocities in the Boltzmann equa-}

tion. As a conclusion, we would like to bring attention to the fact that this question of large velocities and high frequencies is not specific to hydrodynamic limits of the Boltzmann equation. What is actually not clear is the relevance of the Boltzmann 
equation in such regimes. We indeed recall that this equation is expected to model the evolution of rarefied gases and that it has been rigorously derived from Hamiltonian dynamics of elementary particles in the Boltzmann-Grad scaling under very restrictive assumptions.

Actually, these assumptions lead to a short time validity for the full nonlinear Boltzmann equation (see [30] and references therein), which is anyway not compatible with the study of the fast relaxation limit. But we could hope that this restriction is technical. The important point is that, in order to control recollisions (which are not predicted by the Boltzmann equation) and to prove that their occurrence has vanishing probability in the limit, we need to control both concentrations and large velocities. The suitable norms are typically weighted $L^{\infty}$-norms giving some exponential decay for large $v$. The possibility of extending Lanford's arguments to enlarged functional spaces is a challenging question (see [7,8] for instance), but this probably would not be enough to avoid nonlinear pathologies.

This means that we have to think about regularization and renormalization methods, not only for fluid limits but as a more general question regarding gas dynamics.

\section{AbOut THE AUTHOR}

Laure Saint-Raymond is professor at Université Pierre et Marie Curie and at École Normale Supérieure in Paris. She has many important contributions to the study of hydrodynamic limits of the Boltzmann equation, which is part of Hilbert's 6th Problem.

\section{REFERENCES}

[1] Diogo Arsénio and Laure Saint-Raymond, Compactness in kinetic transport equations and hypoellipticity, J. Funct. Anal. 261 (2011), no. 10, 3044-3098, DOI 10.1016/j.jfa.2011.07.020. MR2832590(2012h:35029)

[2] D. Arsénio and L. Saint-Raymond. From the Vlasov-Maxwell-Boltzmann system to incompressible viscous electro-magneto-hydrodynamics. In preparation (2013).

[3] Claude Bardos, François Golse, and C. David Levermore, Fluid dynamic limits of kinetic equations. II. Convergence proofs for the Boltzmann equation, Comm. Pure Appl. Math. 46 (1993), no. 5, 667-753, DOI 10.1002/cpa.3160460503. MR.1213991 (94g:82039)

[4] Claude Bardos and Seiji Ukai, The classical incompressible Navier-Stokes limit of the Boltzmann equation, Math. Models Methods Appl. Sci. 1 (1991), no. 2, 235-257, DOI 10.1142/S0218202591000137. MR1115292 (93a:76023)

[5] A. V. Bobylëv, On the Chapman-Enskog and Grad methods for solving the Boltzmann equation, Dokl. Akad. Nauk SSSR 262 (1982), no. 1, 71-75 (Russian). MR647994 (83g:82034)

[6] A. V. Bobylev, Instabilities in the Chapman-Enskog expansion and hyperbolic Burnett equations, J. Stat. Phys. 124 (2006), no. 2-4, 371-399, DOI 10.1007/s10955-005-8087-6. MR2264613 (2007i:82066)

[7] T. Bodineau, I. Gallagher, and L. Saint-Raymond. The Brownian motion as the limit of a deterministic system of hard spheres. Submitted (2013).

[8] T. Bodineau, I. Gallagher, and L. Saint-Raymond. Linear fluid models as scaling limits of systems of particles. Work in progress (2013).

[9] Stefano Bianchini and Alberto Bressan, Vanishing viscosity solutions of nonlinear hyperbolic systems, Ann. of Math. (2) 161 (2005), no. 1, 223-342, DOI 10.4007/annals.2005.161.223. MR2150387(2007i:35160)

[10] Russel E. Caflisch, The fluid dynamic limit of the nonlinear Boltzmann equation, Comm. Pure Appl. Math. 33 (1980), no. 5, 651-666, DOI 10.1002/cpa.3160330506. MR586416 (81j:76072)

[11] Russel E. Caflisch and Basil Nicolaenko, Shock profile solutions of the Boltzmann equation, Comm. Math. Phys. 86 (1982), no. 2, 161-194. MR676183(84d:82022) 
[12] Sydney Chapman and T. G. Cowling, The mathematical theory of non-uniform gases: An account of the kinetic theory of viscosity, thermal conduction, and diffusion in gases, Cambridge University Press, New York, 1960. MR0116537 (22 \#7324)

[13] Carlo Cercignani, The Boltzmann equation and its applications, Applied Mathematical Sciences, vol. 67, Springer-Verlag, New York, 1988. MR.1313028 (95i:82082)

[14] L. Desvillettes and C. Villani, On the trend to global equilibrium for spatially inhomogeneous kinetic systems: the Boltzmann equation, Invent. Math. 159 (2005), no. 2, 245-316, DOI 10.1007/s00222-004-0389-9. MR2116276 (2005j:82070)

[15] R. J. DiPerna and P.-L. Lions, On the Cauchy problem for Boltzmann equations: global existence and weak stability, Ann. of Math. (2) 130 (1989), no. 2, 321-366, DOI 10.2307/1971423. MR $1014927(90 \mathrm{k}: 82045)$

[16] R. J. DiPerna, P.-L. Lions, and Y. Meyer, $L^{p}$ regularity of velocity averages, Ann. Inst. H. Poincaré Anal. Non Linéaire 8 (1991), no. 3-4, 271-287 (English, with French summary). MR.1127927 (92g:35036)

[17] François Golse, Pierre-Louis Lions, Benoît Perthame, and Rémi Sentis, Regularity of the moments of the solution of a transport equation, J. Funct. Anal. 76 (1988), no. 1, 110-125, DOI 10.1016/0022-1236(88)90051-1. MR923047 (89a:35179)

[18] I. Gallagher, L. Saint-Raymond, and B. Texier. From Newton to Boltzmann: the case of hard-spheres and short-range potentials, to appear in Zürich Lectures Adv. Math. (2013).

[19] F. Golse, D. Levermore, and L. Saint-Raymond. La méthode de l'entropie relative pour les limites hydrodynamiques de modèles cinétiques, Séminaire Equations aux dérivées partielles (Polytechnique) (1999-2000).

[20] François Golse and Laure Saint-Raymond, The Navier-Stokes limit of the Boltzmann equation for bounded collision kernels, Invent. Math. 155 (2004), no. 1, 81-161, DOI 10.1007/s00222-003-0316-5. MR2025302 (2005f:76003)

[21] François Golse and Laure Saint-Raymond, The incompressible Navier-Stokes limit of the Boltzmann equation for hard cutoff potentials, J. Math. Pures Appl. (9) 91 (2009), no. 5, 508-552, DOI 10.1016/j.matpur.2009.01.013 (English, with English and French summaries). MR2517786 (2010j:35378)

[22] François Golse and Laure Saint-Raymond, Velocity averaging in $L^{1}$ for the transport equation, C. R. Math. Acad. Sci. Paris 334 (2002), no. 7, 557-562, DOI 10.1016/S1631-073X(02)023026 (English, with English and French summaries). MR.1903763 (2003f:35044)

[23] Alexander N. Gorban and Iliya V. Karlin, Structure and approximations of the ChapmanEnskog expansion for the linearized Grad equations, Transport Theory Statist. Phys. 21 (1992), no. 1-2, 101-117, DOI 10.1080/00411459208203524. MR1149364 (92m:82117)

[24] Iliya V. Karlin and Alexander N. Gorban, Hydrodynamics from Grad's equations: what can we learn from exact solutions?, Ann. Phys. 11 (2002), no. 10-11, 783-833, DOI 10.1002/15213889(200211)11:10/11<783::AID-ANDP783〉3.0.CO;2-V. MR.1957348 (2004e:82050)

[25] A. K. Gorban and I. Karlin, Hilbert's 6th Problem: exact and approximate hydrodynamic manifolds for kinetic equations Bull. Amer. Math. Soc. 51 (2014), no. 2, 186-246.

[26] Harold Grad, Asymptotic theory of the Boltzmann equation. II, Rarefied Gas Dynamics (Proc. 3rd Internat. Sympos., Palais de l'UNESCO, Paris, 1962), Vol. I, Academic Press, New York, 1963, pp. 26-59. MR0156656 (27 \#6577)

[27] E. Grenier, Quelques limites singulières oscillantes, Séminaire sur les Équations aux Dérivées Partielles, 1994-1995, École Polytech., Palaiseau, 1995, pp. Exp. No. XXI, 13 (French). MR:1362569 (96m:82076)

[28] E. H. Hauge, Exact and Chapman-Enskog solutions of the Boltzmann equation for the Lorentz model, Phys. Fluids 13 (1970), 1201-1208. MR0278672 (43 \#4402)

[29] David Hilbert, Begründung der kinetischen Gastheorie, Math. Ann. 72 (1912), no. 4, 562-577, DOI 10.1007/BF01456676 (German). MR:1511713

[30] Oscar E. Lanford III, Time evolution of large classical systems, Dynamical systems, theory and applications (Recontres, Battelle Res. Inst., Seattle, Wash., 1974), Springer, Berlin, 1975, pp. 1-111. Lecture Notes in Phys., Vol. 38. MR0479206 (57 \#18653)

[31] J. Leray. Etude de diverses équations intégrales non linéaires et quelques problèmes que pose l'hydrodynamique, J. Math. Pures Appl. 9 (1933), 1-82.

[32] P.-L. Lions, Compactness in Boltzmann's equation via Fourier integral operators and applications J. Math. Kyoto Univ. 34 (1994), 391-427, 429-461, 539-584. 
[33] Pierre-Louis Lions, Mathematical topics in fluid mechanics. Vol. 1, Oxford Lecture Series in Mathematics and its Applications, vol. 3, The Clarendon Press Oxford University Press, New York, 1996. Incompressible models; Oxford Science Publications. MR1422251 (98b:76001)

[34] Pierre-Louis Lions and Nader Masmoudi, Une approche locale de la limite incompressible, C. R. Acad. Sci. Paris Sér. I Math. 329 (1999), no. 5, 387-392, DOI 10.1016/S07644442(00)88611-5 (French, with English and French summaries). MR.1710123 (2000e:76103)

[35] Tai Ping Liu, Solutions in the large for the equations of nonisentropic gas dynamics, Indiana Univ. Math. J. 26 (1977), no. 1, 147-177. MR0435618 (55 \#8576)

[36] Tai-Ping Liu and Shih-Hsien Yu, Boltzmann equation: micro-macro decompositions and positivity of shock profiles, Comm. Math. Phys. 246 (2004), no. 1, 133-179, DOI 10.1007/s00220003-1030-2. MR2044894 (2005f:82101)

[37] Laure Saint-Raymond, Convergence of solutions to the Boltzmann equation in the incompressible Euler limit, Arch. Ration. Mech. Anal. 166 (2003), no. 1, 47-80, DOI 10.1007/s00205-002-0228-3. MR.1952079 (2004d:35038)

[38] Laure Saint-Raymond, Hydrodynamic limits: some improvements of the relative entropy method, Ann. Inst. H. Poincaré Anal. Non Linéaire 26 (2009), no. 3, 705-744, DOI 10.1016/j.anihpc.2008.01.001 (English, with English and French summaries). MR2526399 (2010g:76028)

[39] Laure Saint-Raymond, Hydrodynamic limits of the Boltzmann equation, Lecture Notes in Mathematics, vol. 1971, Springer-Verlag, Berlin, 2009. MR2683475 (2012f:82079)

[40] Steven Schochet, Fast singular limits of hyperbolic PDEs, J. Differential Equations 114 (1994), no. 2, 476-512, DOI 10.1006/jdeq.1994.1157. MR1303036 (95k:35131)

[41] Thomas C. Sideris, Formation of singularities in three-dimensional compressible fluids, Comm. Math. Phys. 101 (1985), no. 4, 475-485. MR815196 (87d:35127)

[42] M. Slemrod. Admissibility of weak solutions for the compressible Euler equations, $n \geq 2$. To appear in Philosophical Transactions of the Royal Society A (2013).

[43] Horng-Tzer Yau, Relative entropy and hydrodynamics of Ginzburg-Landau models, Lett. Math. Phys. 22 (1991), no. 1, 63-80, DOI 10.1007/BF00400379. MR.1121850 (93e:82035)

Département de Mathématiques et Applications, École Normale Supérieure, Paris, FRANCE

Current address: 45 rue d'Ulm, 75230 Paris Cedex 05, France

E-mail address: Laure.Saint-Raymond@ens.fr 